\title{
GROWTH PROPERTIES OF POTENTIALS IN CENTRAL MORREY-ORLICZ SPACES ON THE UNIT BALL
}

\author{
Yoshihiro Mizuta, Takao Ohno and Tetsu Shimomura \\ 4-13-11 Hachi-Hon-Matsu-Minami, Higashi-Hiroshima 739-0144, Japan; \\ yomizuta@hiroshima-u.ac.jp \\ Oita University, Faculty of Education \\ Dannoharu Oita-city 870-1192, Japan; t-ohno@oita-u.ac.jp \\ Hiroshima University, Graduate School of Education, Department of Mathematics \\ Higashi-Hiroshima 739-8524, Japan; tshimo@hiroshima-u.ac.jp
}

\begin{abstract}
We introduce central Morrey-Orlicz spaces $M^{\Phi, \omega}(\mathbf{B})$ on the unit ball and study the existence of weighted spherical limits:

$$
\liminf _{r \rightarrow 1-}(1-r)^{d_{1}} \omega(1-r)^{d_{2}}\left(\int_{S(0, r)} \Phi\left((1-r)^{d_{3}}\left|I_{\alpha} f(x)\right|\right)^{q} d S(x)\right)^{1 / q}
$$

for some $d_{1}, d_{2}, d_{3} \in \mathbf{R}, 1 \leq q<\infty$, and all Riesz potentials $I_{\alpha} f$ with $f \in M^{\Phi, \omega}(\mathbf{B})$. We also deal with the existence of weighted spherical limits for Green potentials and monotone Sobolev functions.
\end{abstract}

\section{Introduction}

Let $\mathbf{R}^{N}, N \geq 2$, denote the $N$-dimensional Euclidean space. We use the notation $B(x, r)$ to denote the open ball centered at $x$ with radius $r>0$, whose boundary is denoted by $S(x, r)$. The $L^{q}$ means over the spherical surface $S(0, r)$ for $u$ is defined by

$$
S_{q}(u, r)=\left(\frac{1}{|S(0, r)|} \int_{S(0, r)}|u(x)|^{q} d S(x)\right)^{1 / q}=\left(\frac{1}{\omega_{N-1}} \int_{S(0,1)}|u(r \sigma)|^{q} d S(\sigma)\right)^{1 / q}
$$

when $1 \leq q<\infty$, where $|S(0, r)|=\omega_{N-1} r^{N-1}$ with $\omega_{N-1}$ being the area of the unit sphere. Gardiner [4, Theorem 2] showed that

$$
\liminf _{r \rightarrow 1-}(1-r)^{(N-1)(1-1 / q)} S_{q}(u, r)=0
$$

when $u$ is a Green potential in the unit ball $\mathbf{B}=B(0,1),(N-3) /(N-1)<1 / q \leq$ $(N-2) /(N-1)$ and $q>0$, as an extension of the result by Stoll [21] in the plane case. In [12], The first author gave versions of Gardiner's result in [4] to the half space. The first and third authors [17] studied the existence of boundary limits for BLD (Beppo Levi and Deny) functions $u$ on the unit ball $\mathbf{B}$ of $\mathbf{R}^{N}$ satisfying

$$
\int_{\mathbf{B}}|\nabla u(x)|^{p}(1-|x|)^{\gamma} d x<\infty
$$

where $\nabla$ denotes the gradient, $1<p<\infty$ and $-1<\gamma<p-1$. In fact, we showed that

$$
\liminf _{r \rightarrow 1-}(1-r)^{(N-p+\gamma) / p-(N-1) / q} S_{q}(u, r)=0
$$

https://doi.org/10.5186/aasfm.2018.4302

2010 Mathematics Subject Classification: Primary 31B15, 46E35.

Key words: Spherical limits, central Morrey-Orlicz spaces, Riesz potentials, Green potentials. 
when $q>0$ and $(N-p-1) /(p(N-1))<1 / q<(N-p+\gamma) /(p(N-1))$, as a result corresponding to [16, Theorem 2.1] given in half spaces. In [17], we also studied the existence of boundary limits for monotone BLD functions $u$ on the unit ball $\mathbf{B}$ of $\mathbf{R}^{N}$ satisfying (1.1).

We denote by $M^{\Phi, \omega}(\mathbf{B})$ the class of measurable functions $f$ on the unit ball $\mathbf{B}$ satisfying

$$
\|f\|_{M^{\Phi, \omega}(\mathbf{B})}=\sup _{0<r<1} \omega(1-r)\|f\|_{L^{\Phi}(\mathbf{B} \backslash B(0, r))}<\infty
$$

with a convex function $\Phi$ and a doubling weight $\omega$; the space $M^{\Phi, \omega}(\mathbf{B})$ is referred to as a central Morrey-Orlicz space (see Section 2 for the definitions of $\Phi$ and $\omega$ ). For these spaces, see e.g. [1, 2, 3, 18]. When $\Phi(r)=r^{p}$ and $\gamma<0$, one can find $u$ such that $|\nabla u| \in M^{\Phi, \omega}(\mathbf{B})$ but $u$ does not satisfy (1.1); see also Remark 3.5.

For $0<\alpha<N$, we define the Riesz potential of order $\alpha$ for locally integrable function $f$ on $\mathbf{B}$ by

$$
I_{\alpha} f(x)=\int_{\mathbf{B}}|x-y|^{\alpha-N} f(y) d y .
$$

Our main aim in this paper is to discuss the weighted limit

$$
(1-r)^{d_{1}} \omega(1-r)^{d_{2}} S_{q}\left(\Phi\left((1-r)^{d_{3}} I_{\alpha} f\right), r\right)
$$

as $r \rightarrow 1-0$ for $I_{\alpha} f$ with $f \in M^{\Phi, \omega}(\mathbf{B}) ; d_{1}, d_{2}$ and $d_{3}$ will be given later (see Theorem 3.1 below). The result is new even for $M^{p, \nu}(\mathbf{B})$, that is, for the case $\Phi(r)=$ $r^{p}$ and $\omega(r)=r^{-\nu}$. The sharpness of the exponent of $1-r$ will be discussed later.

In Section 4, as an application of Theorem 3.1, we treat functions $f$ on $\mathbf{B}$ satisfying the weighted condition $(1-|y|)^{\beta p_{1}} f(y)^{p_{1}} \in M^{\Phi, \omega}(\mathbf{B})$ for $1<p_{1}<\infty$ and $\beta>0$ (see Theorem 4.2 below).

Let $G(x, y)$ be a Green kernel on B. We define the Green potential for locally integrable function $f$ on $\mathbf{B}$ by

$$
G f(x)=\int_{\mathbf{B}} G(x, y) f(y) d y .
$$

In Section 5, we study the existence of weighted spherical limits for Green potentials $G f$ with $(1-|y|) f(y) \in M^{\Phi, \omega}(\mathbf{B})$ in our settings (see Theorem 5.3 below).

A continuous function $u$ on an open set $\Omega$ is called monotone in the sense of Lebesgue [7] if for every relatively compact open set $G \subset \Omega$,

$$
\max _{\bar{G}} u=\max _{\partial G} u \quad \text { and } \quad \min _{\bar{G}} u=\min _{\partial G} u .
$$

Harmonic functions on $\Omega$ are monotone in $\Omega$. More generally, solutions of elliptic partial differential equations of second order and weak solutions for variational problems may be monotone (see [5]). See also [6], [9], [10], [14], [15], [24], [25] and [26].

In the last section, we study the existence of weighted spherical limits for monotone functions $u$ with $|\nabla u(y)|^{p_{1}} \in M^{\Phi, \omega}(\mathbf{B})$ with $p_{1}>N-1$ in our settings (see Theorem 6.1 below). Essential tool in treating monotone functions is Lemma 6.2 below.

For related results on spherical means, see [11], [13], [15], [20], [22] and [23]. We also refer the reader to the papers [8] and [19] for weighted integral means over balls.

\section{Preliminaries and lemmas}

Throughout this paper, let $C$ denote various positive constants independent of the variables in question. The symbol $g \sim h$ means that $C^{-1} h \leq g \leq C h$ for some constant $C>0$. 
Let $\Phi$ be a convex function on $[0, \infty)$ such that

$(\Phi 1) \Phi(0)=0$ and $\Phi(r)>0$ for $r>0$;

$(\Phi 2) \Phi$ is doubling, that is, there exists a constant $A_{1}>0$ such that

$$
\Phi(2 r) \leq A_{1} \Phi(r) \text { for } r>0 ;
$$

(Ф3) for some $p \geq 1, r^{-p} \Phi(r)$ is almost increasing, that is, there exists a constant $A_{2}>0$ such that

$$
\Phi(r t) \leq A_{2} r^{p} \Phi(t) \quad \text { when } 0<r<1 \text { and } t>0 .
$$

Further consider a weight $\omega$ such that

$(\omega 1) \omega(r)>0$ for $r>0$;

$(\omega 2) \omega$ is almost decreasing in $(0, \infty)$, that is, there is a constant $C>0$ such that

$$
\omega(t) \leq C \omega(s) \text { when } 0<s<t<\infty
$$

$(\omega 3) \omega$ is doubling.

We see that $\omega(r)=r^{-\nu}\left(\log \left(e+r^{-1}\right)\right)^{\tau}$ is almost decreasing when $\nu>0$ and $\tau \in \mathbf{R}$.

Note here that (\$3) holds if and only if

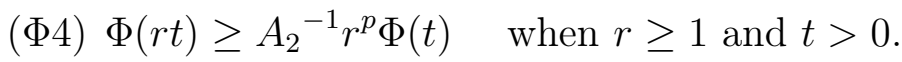

Moreover, if $\Phi$ is of the form $r^{p_{1}}(\log (e+r))^{\theta}$, then $(\Phi 3)$ holds when $p_{1}>p$ or when $p_{1}=p$ and $\theta \geq 0$.

For an open set $G$ in $\mathbf{R}^{N}$, we define the Luxemburg-Nakano-Orlicz norm for $f \in L_{\mathrm{loc}}^{1}(G)$ by

$$
\|f\|_{L^{\Phi}(G)}=\inf \left\{\lambda>0: \int_{G} \Phi(|f(y)| / \lambda) d y \leq 1\right\}
$$

we set $f=0$ outside $G$ for the sake of convenience.

We consider the family $M^{\Phi, \omega}(\mathbf{B})$ of all measurable functions $f$ on $\mathbf{B}$ satisfying

$$
\|f\|_{M^{\Phi, \omega}(\mathbf{B})}=\sup _{0<r<1} \omega(1-r)\|f\|_{L^{\Phi}(\mathbf{B} \backslash B(0, r))}<\infty .
$$

When $\Phi(r)=r^{p}$ and $\omega(r)=r^{-\nu}, M^{\Phi, \omega}(\mathbf{B})$ will be written as $M^{p, \nu}(\mathbf{B})$. It is easy to see that

$$
\sup _{0<r<1} \omega(1-r)\|f\|_{L^{\Phi}(\mathbf{B} \backslash B(0, r))}<\infty
$$

if and only if

$$
\sup _{0<r<1} \int_{\mathbf{B} \backslash B(0, r)} \Phi(\omega(1-r)|f(y)|) d y<\infty .
$$

Moreover it is useful to note the following result.

Lemma 2.1. Let $\Phi(r)=r^{p}(\log (c+r))^{\theta}$ and $\omega(r)=r^{-\nu}(\log (c+r))^{\tau}$ for $p>1$ and real numbers $\theta, \nu, \tau$, where $c>1$ is chosen so large that $\Phi$ is convex. If $0 \leq \nu<1 / p$, then the following are equivalent:

(1) there exists a constant $C_{1}>0$ such that

$$
\sup _{0<r<1} \omega(1-r)\|f\|_{L^{\Phi}(\mathbf{B} \backslash B(0, r))} \leq C_{1} ;
$$

(2) there exists a constant $C_{2}>0$ such that

$$
\sup _{0<r<1} \omega(1-r)^{p} \int_{\mathbf{B} \backslash B(0, r)} \Phi(|f(y)|) d y \leq C_{2} ;
$$

here $C_{1} \sim C_{2}$. 
Proof. We treat only the case when $\theta \geq 0$, since the case $\theta<0$ is similarly treated. Let $0<r<1$ and $t>0$. By $(\Phi 4)$ and $(\omega 2)$, we have

$$
\omega(1-r)^{p} \Phi(t) \leq C \Phi(\omega(1-r) t),
$$

so that (1) implies (2). Next, if $\omega(1-r) t \leq t^{1+A}$ for $A>0$, then

$$
\begin{aligned}
\Phi(\omega(1-r) t) & =(\omega(1-r) t)^{p}(\log (c+\omega(1-r) t))^{\theta} \\
& \leq(\omega(1-r) t)^{p}\left(\log \left(c+t^{1+A}\right)\right)^{\theta} \\
& \leq C \omega(1-r)^{p} t^{p}(\log (c+t))^{\theta}=C \omega(1-r)^{p} \Phi(t)
\end{aligned}
$$

and if $\omega(1-r) t>t^{1+A}$, then $t \leq \omega(1-r)^{1 / A}$, so that

$$
\Phi(\omega(1-r) t) \leq \Phi\left(\omega(1-r)^{1+1 / A}\right) .
$$

Hence

$$
\Phi(\omega(1-r) t) \leq C\left\{\omega(1-r)^{p} \Phi(t)+\Phi\left(\omega(1-r)^{1+1 / A}\right)\right\} .
$$

If $\nu p<1$, then we note that

$$
\int_{\mathbf{B} \backslash B(0, r)} \Phi\left(\omega(1-r)^{1+1 / A}\right) d y=C(1-r) \Phi\left(\omega(1-r)^{1+1 / A}\right)<\infty,
$$

when $A$ is so large that $\nu p(1+1 / A)<1$. Now the equivalence of assertions (1) and (2) is obtained.

Here we give an estimate for spherical means for Riesz kernels.

Lemma 2.2. Let $0<a<N$ and $c_{1}, c_{2}$ be positive constants. If $c_{1}|y|<t<c_{2}|y|$ and $1 / 2<|y|<1$, then there exists a constant $C>0$ such that

$$
\int_{S(0,1) \cap B(y, 1-t)}|t \sigma-y|^{a-N} d S(\sigma) \leq C \begin{cases}|t-| y||^{a-1} & \text { when } a<1 ; \\ (1-t)^{a-1} & \text { when } a>1 .\end{cases}
$$

Proof. By an application of polar coordinates, we note that

$$
\begin{aligned}
& \int_{S(0,1) \cap B(y, 1-t)}|t \sigma-y|^{a-N} d S(\sigma) \\
& \leq C \int_{0}^{\sin ^{-1} 2(1-t)}|| y\left|+t^{2}-2\right| y|t \cos \theta|^{(a-N) / 2} \sin ^{N-2} \theta d \theta \\
& \leq C \int_{0}^{c(1-t)}\left(|| y|-t|^{2}+t^{2} \theta^{2}\right)^{(a-N) / 2} \theta^{N-2} d \theta \\
& =C t^{1-N}|| y|-t|^{a-1} \int_{0}^{c(1-t) t /|y|-t \mid}\left(1+s^{2}\right)^{(a-N) / 2} s^{N-2} d s \\
& \leq C t^{1-N}|| y|-t|^{a-1} \times \begin{cases}1 & \text { when } a<1, \\
((1-t) t /|| y|-t|)^{a-1} & \text { when } a>1 .\end{cases}
\end{aligned}
$$

Thus the present lemma is obtained.

Lemma 2.3. Let $0<a<N$ and $c_{1}, c_{2}$ be positive constants. If $c_{1}|y|<t<c_{2}|y|$ and $1 / 2<|y|<1$, then there exists a constant $C>0$ such that

$$
\int_{S(0,1)}|t \sigma-y|^{a-N} d S(\sigma) \geq C \begin{cases}|t-| y||^{a-1} & \text { when } a<1 ; \\ 1 & \text { when } a>1 .\end{cases}
$$


Proof. By an application of polar coordinates, we have

$$
\begin{aligned}
\int_{S(0,1)}|t \sigma-y|^{a-N} d S(\sigma) & =C \int_{0}^{\pi}|| y\left|+t^{2}-2\right| y|t \cos \theta|^{(a-N) / 2} \sin ^{N-2} \theta d \theta \\
& \geq C \int_{0}^{\pi / 2}\left(|| y|-t|^{2}+t^{2} \theta^{2}\right)^{(a-N) / 2} \theta^{N-2} d \theta \\
& =C t^{1-N}|| y|-t|^{a-1} \int_{0}^{\pi t /(2|| y|-t|)}\left(1+s^{2}\right)^{(a-N) / 2} s^{N-2} d s \\
& \geq C t^{1-N}|| y|-t|^{a-1} \times \begin{cases}1 & \text { when } a<1, \\
(\pi t /|| y|-t|)^{a-1} & \text { when } a>1\end{cases}
\end{aligned}
$$

since

$$
\pi t /(2|| y|-t|) \geq \pi t /(2(|y|+t)) \geq \pi c_{1} /\left(2\left(1+c_{1}\right)\right)>0
$$

when $c_{1}|y|<t<c_{2}|y|$. Thus the present lemma is obtained.

For a nonnegative function $f \in L_{\text {loc }}^{1}(\mathbf{B})$ and $x \in \mathbf{B}$, write

$$
\begin{aligned}
I_{\alpha} f(x)= & \int_{B(x,(1-|x|) / 2)}|x-y|^{\alpha-N} f(y) d y \\
& +\int_{\{y \in \mathbf{B} \backslash B(x,(1-|x|) / 2): 1-|y| \leq 1-|x|\}}|x-y|^{\alpha-N} f(y) d y \\
& +\int_{\{y \in \mathbf{B} \backslash B(x,(1-|x|) / 2): 1-|y|>1-|x|\}}|x-y|^{\alpha-N} f(y) d y \\
= & I_{1}(x)+I_{2}(x)+I_{3}(x) .
\end{aligned}
$$

Set

$$
A(0, r)=B(0, r+(1-r) / 2) \backslash B(0, r-(1-r) / 2) .
$$

Lemma 2.4. Let $1 \leq q<\infty$.

(1) Suppose $0<\varepsilon<\alpha$ and

$$
(N-1) / q<N-\alpha p+\varepsilon(p-1) .
$$

Then there exists a constant $C>0$ such that

$$
\begin{aligned}
& S_{q}\left(\Phi\left((1-r)^{-\varepsilon} \omega(1-r) I_{1}\right), r\right) \\
& \leq C(1-r)^{-\varepsilon} \int_{A(0, r)}|r-| y||^{(\alpha-\varepsilon) p+\varepsilon-N+(N-1) / q} \Phi(\omega(1-r) f(y)) d y
\end{aligned}
$$

for all $1 / 2<r<1$ and nonnegative measurable functions $f \in L_{\mathrm{loc}}^{1}(\mathbf{B})$.

(2) Suppose $0<\varepsilon<\alpha$ and

$$
(N-1) / q>N-\alpha p+\varepsilon(p-1)>0 .
$$

Then there exists a constant $C>0$ such that

$$
S_{q}\left(\Phi\left((1-r)^{-\varepsilon} \omega(1-r) I_{1}\right), r\right) \leq C(1-r)^{(\alpha-\varepsilon) p-N+(N-1) / q}
$$

for all $1 / 2<r<1$ and nonnegative measurable functions $f$ on $\mathbf{B}$ with $\|f\|_{M^{\Phi, \omega}(\mathbf{B})} \leq 1$.

(3) Suppose $0<\varepsilon<\alpha$ and $(\alpha-\varepsilon) p+\varepsilon-N>0$. Then there exists a constant $C>0$ such that

$$
\Phi\left((1-r)^{-\varepsilon} \omega(1-r) I_{1}\right) \leq C(1-r)^{(\alpha-\varepsilon) p-N}
$$


for all $1 / 2<r<1$ and nonnegative measurable functions $f$ on $\mathbf{B}$ with $\|f\|_{M^{\Phi, \omega}(\mathbf{B})} \leq 1$.

Proof. Let $0<\varepsilon<\alpha$ and

$$
(\alpha-\varepsilon) p+\varepsilon-N+(N-1) / q<0 .
$$

For $1 / 2<r=|x|<1$, we have

$$
\begin{aligned}
I_{1}(x) & =\int_{B(x,(1-|x|) / 2)}|x-y|^{\alpha-N} f(y) d y \\
& \leq C \int_{0}^{1-r}\left(\frac{1}{|B(x, t)|} \int_{B(x, t) \cap A(0, r)} f(y) d y\right) t^{\alpha-1} d t \\
& \leq C \int_{0}^{1-r}\left(\frac{1}{|B(x, t)|} \int_{B(x, t) \cap A(0, r)} t^{\alpha-\varepsilon} f(y) d y\right) t^{\varepsilon-1} d t
\end{aligned}
$$

since $B(x,(1-|x|) / 2) \subset A(0, r)$, where $|B|$ denotes the volume of balls $B$.

We have by Jensen's inequality and $(\Phi 3)$

$$
\begin{aligned}
& \Phi\left((1-r)^{-\varepsilon} \omega(1-r) I_{1}(x)\right) \\
& \leq C \Phi\left((1-r)^{-\varepsilon} \omega(1-r) \int_{0}^{1-r}\left(\frac{1}{|B(x, t)|} \int_{B(x, t) \cap A(0, r)} t^{\alpha-\varepsilon} f(y) d y\right) t^{\varepsilon-1} d t\right) \\
& \leq C(1-r)^{-\varepsilon} \int_{0}^{1-r}\left(\frac{1}{|B(x, t)|} \int_{B(x, t) \cap A(0, r)} \Phi\left(t^{\alpha-\varepsilon} \omega(1-r) f(y)\right) d y\right) t^{\varepsilon-1} d t \\
& \leq C(1-r)^{-\varepsilon} \int_{0}^{1-r} t^{(\alpha-\varepsilon) p-N}\left(\int_{B(x, t) \cap A(0, r)} \Phi(\omega(1-r) f(y)) d y\right) t^{\varepsilon-1} d t \\
& \leq C(1-r)^{-\varepsilon} \int_{A(0, r)}|x-y|^{(\alpha-\varepsilon) p+\varepsilon-N} \Phi(\omega(1-r) f(y)) d y
\end{aligned}
$$

since $(\alpha-\varepsilon) p+\varepsilon-N<0$.

Hence in this case Minkowski's inequality and Lemma 2.2 yield

$$
\begin{aligned}
& S_{q}\left(\Phi\left((1-r)^{-\varepsilon} \omega(1-r) I_{1}\right), r\right) \\
& \leq C(1-r)^{-\varepsilon} \int_{A(0, r)} S_{q}\left(|\cdot-y|^{(\alpha-\varepsilon) p+\varepsilon-N}, r\right) \Phi(\omega(1-r) f(y)) d y \\
& \leq\left. C(1-r)^{-\varepsilon} \int_{A(0, r)}|r-| y\right|^{(\alpha-\varepsilon) p+\varepsilon-N+(N-1) / q} \Phi(\omega(1-r) f(y)) d y
\end{aligned}
$$

since $(\alpha-\varepsilon) p+\varepsilon-N+(N-1) / q<0,1 / 2<r<1$ and $r \sim|y|$ on $A(0, r)$, which gives assertion (1). above

Next we shall show assertion (2). Similarly, under our assumptions, we obtain as

$$
\begin{aligned}
& S_{q}\left(\Phi\left((1-r)^{-\varepsilon} \omega(1-r) I_{1}\right), r\right) \\
& \leq C(1-r)^{-\varepsilon} \int_{A(0, r)} S_{q}\left(|\cdot-y|^{(\alpha-\varepsilon) p+\varepsilon-N} \chi_{B(y,(1-r) / 2)}, r\right) \Phi(\omega(1-r) f(y)) d y \\
& \leq C(1-r)^{-\varepsilon}(1-r)^{(\alpha-\varepsilon) p+\varepsilon-N+(N-1) / q} \int_{A(0, r)} \Phi(\omega(1-r) f(y)) d y \\
& \leq C(1-r)^{(\alpha-\varepsilon) p-N+(N-1) / q}
\end{aligned}
$$


for $1 / 2<r<1$, since

$$
\begin{aligned}
\int_{A(0, r)} \Phi(\omega(1-r) f(y)) d y & \leq \int_{\mathbf{B} \backslash B(0, s)} \Phi(\omega(2(1-s) / 3) f(y)) d y \\
& \leq C \int_{\mathbf{B} \backslash B(0, s)} \Phi(\omega(1-s) f(y)) d y \leq C,
\end{aligned}
$$

where $s=r-(1-r) / 2$. Thus assertion (2) is proved.

Finally we shall show assertion (3). When $0<\varepsilon<\alpha$ and $(\alpha-\varepsilon) p+\varepsilon-N>0$, we have

$$
\begin{aligned}
& \Phi\left((1-r)^{-\varepsilon} \omega(1-r) I_{1}(x)\right) \\
& \leq C(1-r)^{-\varepsilon} \int_{0}^{1-r} t^{(\alpha-\varepsilon) p-N}\left(\int_{B(x, t) \cap A(0, r)} \Phi(\omega(1-r) f(y)) d y\right) t^{\varepsilon-1} d t \\
& \leq C(1-r)^{-\varepsilon}(1-r)^{(\alpha-\varepsilon) p+\varepsilon-N} \int_{A(0, r)} \Phi(\omega(1-r) f(y)) d y \leq C(1-r)^{(\alpha-\varepsilon) p-N},
\end{aligned}
$$

which proves assertion (3).

Lemma 2.5. Let $0<d<1$ and $M>0$. Set

$$
G(t)=(1-t)^{d} \int_{A(0, t)}|t-| y||^{-d} g(y) d y
$$

for a nonnegative measurable function $g$ such that $\sup _{0<t<1} \int_{A(0, t)} g(y) d y \leq M$. Then there exists a constant $c>0$ such that

$$
\inf _{1-2^{-j+1}<t<1-2^{-j}} G(t)<c M \quad \text { for each positive integer } j \text {. }
$$

Proof. For each positive integer $j$, we have

$$
\begin{aligned}
& \int_{1-2^{-j+1}}^{1-2^{-j}} G(t) \frac{d t}{1-t} \\
& \leq C \int_{A\left(0,1-2^{-j}\right) \cup A\left(0,1-2^{-j+1}\right)}\left(2^{-j(d-1)} \int_{1-2^{-j+1}}^{1-2^{-j}}|t-| y||^{-d} d t\right) g(y) d y \\
& \leq C \int_{A\left(0,1-2^{-j}\right) \cup A\left(0,1-2^{-j+1}\right)} g(y) d y \leq C M .
\end{aligned}
$$

Hence

as required.

$$
\inf _{1-2^{-j+1}<t<1-2^{-j}} G(t) \leq C M /(\log 2),
$$

Lemma 2.6. Let $1 \leq q<\infty$.

(1) Suppose $\varepsilon>0$ and

$$
(N-1) / q<N-\alpha p-\varepsilon(p-1) .
$$

Then there exists a constant $C>0$ such that

$$
S_{q}\left(\Phi\left(\omega(1-r)(1-r)^{\varepsilon} I_{2}\right), r\right) \leq C(1-r)^{(\alpha+\varepsilon) p-N+(N-1) / q}
$$

for all $1 / 2<r<1$ and nonnegative measurable functions $f$ on $\mathbf{B}$ with $\|f\|_{M^{\Phi, \omega}(\mathbf{B})} \leq 1$. 
(2) Suppose $\varepsilon>0$ and

$$
(N-1) / q>N-\alpha p-\varepsilon(p-1) .
$$

Then there exists a constant $C>0$ such that

$$
S_{q}\left(\Phi\left(\omega(1-r)(1-r)^{\varepsilon} I_{2}\right), r\right) \leq C(1-r)^{\varepsilon}
$$

for all $1 / 2<r<1$ and nonnegative measurable functions $f$ on $\mathbf{B}$ with $\|f\|_{M^{\Phi, \omega}(\mathbf{B})} \leq 1$.

Proof. Let $\varepsilon>0$ such that

$$
(N-1) / q<N-(\alpha+\varepsilon) p+\varepsilon .
$$

For $1 / 2<r=|x|<1$, we have

$$
\begin{aligned}
I_{2}(x) & =\int_{\mathbf{B}}|x-y|^{\alpha-N} f_{2, x}(y) d y \\
& \leq C \int_{(1-|x|) / 2}^{2}\left(\frac{1}{|B(x, t)|} \int_{B(x, t)} f_{2, x}(y) d y\right) t^{\alpha-1} d t \\
& \leq C \int_{(1-|x|) / 2}^{2}\left(\frac{1}{|B(x, t)|} \int_{B(x, t)} t^{\alpha+\varepsilon} f_{2, x}(y) d y\right) t^{-\varepsilon-1} d t
\end{aligned}
$$

where $f_{2, x}(y)=f(y) \chi_{E_{2, x}}(y)$ with $E_{2, x}=\{y \in \mathbf{B} \backslash B(x,(1-|x|) / 2): 1-|y| \leq 1-|x|\}$.

We have by Jensen's inequality and $(\Phi 3)$

$$
\begin{aligned}
& \Phi\left(\omega(1-|x|)(1-|x|)^{\varepsilon} I_{2}(x)\right) \\
& \leq C \Phi\left(\omega(1-|x|)(1-|x|)^{\varepsilon} \int_{(1-|x|) / 2}^{2}\left(\frac{1}{|B(x, t)|} \int_{B(x, t)} t^{\alpha+\varepsilon} f_{2, x}(y) d y\right) t^{-\varepsilon-1} d t\right) \\
& \leq C(1-|x|)^{\varepsilon} \int_{(1-|x|) / 2}^{2}\left(\frac{1}{|B(x, t)|} \int_{B(x, t)} \Phi\left(t^{\alpha+\varepsilon} \omega(1-|x|) f_{2, x}(y)\right) d y\right) t^{-\varepsilon-1} d t \\
& \leq C(1-|x|)^{\varepsilon} \int_{(1-|x|) / 2}^{2} t^{(\alpha+\varepsilon) p}\left(\frac{1}{|B(x, t)|} \int_{B(x, t)} \Phi\left(\omega(1-|x|) f_{2, x}(y)\right) d y\right) t^{-\varepsilon-1} d t \\
& \leq C(1-|x|)^{\varepsilon} \int_{\mathbf{B}}|x-y|^{(\alpha+\varepsilon) p-\varepsilon-N} \Phi\left(\omega(1-|x|) f_{2, x}(y)\right) d y
\end{aligned}
$$

since $(\alpha+\varepsilon) p-\varepsilon-N<0$.

By Lemma 2.2, we see that

$$
\begin{aligned}
& \int_{\{\sigma \in S(0,1):|t \sigma-y|>(1-t) / 2\}}|t \sigma-y|^{a-N} d S(\sigma) \\
& \leq \int_{\{\sigma \in S(0,1):|t \sigma-y|>(1-t) / 2\}}(C|(1+(1-t)) \sigma-y|)^{a-N} d S(\sigma) \\
& \leq\left. C|(1+(1-t))-| y\right|^{a-1} \leq C|1-t|^{a-1}
\end{aligned}
$$


for $1 / 2<t<1$ and $y \in \mathbf{B}$, when $a<1$. Hence Minkowski's inequality yields

$$
\begin{aligned}
& S_{q}\left(\Phi\left(\omega(1-r)(1-r)^{\varepsilon} I_{2}\right), r\right) \\
& \leq C(1-r)^{\varepsilon} \int_{\mathbf{B}} S_{q}\left(|\cdot-y|^{(\alpha+\varepsilon) p-\varepsilon-N} \chi_{E_{2, x}}(y), r\right) \Phi(\omega(1-|x|) f(y)) d y \\
& \leq C(1-r)^{\varepsilon}(1-r)^{(\alpha+\varepsilon) p-\varepsilon-N+(N-1) / q} \int_{\mathbf{B} \backslash B(0, r)} \Phi(\omega(1-r) f(y)) d y \\
& \leq C(1-r)^{(\alpha+\varepsilon) p-N+(N-1) / q}
\end{aligned}
$$

since $(\alpha+\varepsilon) p-\varepsilon-N+(N-1) / q<0$, which gives assertion (1).

Next we shall show assertion (2). Suppose $\varepsilon>0$ such that

$$
(N-1) / q>N-(\alpha+\varepsilon) p+\varepsilon>0 .
$$

Then we have by Jensen's inequality and $(\Phi 3)$

$$
\begin{aligned}
& \Phi\left(\omega(1-|x|)(1-|x|)^{\varepsilon} I_{2}(x)\right) \\
& \leq C \Phi\left(\omega(1-|x|)(1-|x|)^{\varepsilon} \int_{(1-|x|) / 2}^{2}\left(\frac{1}{|B(x, t)|} \int_{B(x, t)} t^{\alpha+\varepsilon} f_{2, x}(y) d y\right) t^{-\varepsilon-1} d t\right) \\
& \leq C(1-|x|)^{\varepsilon} \int_{(1-|x|) / 2}^{2}\left(\frac{1}{|B(x, t)|} \int_{B(x, t)} \Phi\left(t^{\alpha+\varepsilon} \omega(1-|x|) f_{2, x}(y)\right) d y\right) t^{-\varepsilon-1} d t \\
& \leq C(1-|x|)^{\varepsilon} \int_{(1-|x|) / 2}^{2} t^{(\alpha+\varepsilon) p}\left(\frac{1}{|B(x, t)|} \int_{B(x, t)} \Phi\left(\omega(1-|x|) f_{2, x}(y)\right) d y\right) t^{-\varepsilon-1} d t \\
& \leq C(1-|x|)^{\varepsilon} \int_{\mathbf{B}}^{|x-y|^{(\alpha+\varepsilon) p-\varepsilon-N} \Phi\left(\omega(1-|x|) f_{2, x}(y)\right) d y .}
\end{aligned}
$$

By Lemma 2.2 and Minkowski's inequality, we find

$$
\begin{aligned}
& S_{q}\left(\Phi\left(\omega(1-r)(1-r)^{\varepsilon} I_{2}\right), r\right) \\
& \leq C(1-r)^{\varepsilon} \int_{\mathbf{B}} S_{q}\left(|\cdot-y|^{(\alpha+\varepsilon) p-\varepsilon-N} \chi_{E_{2, x}}(y), r\right) \Phi(\omega(1-r) f(y)) d y \\
& \leq C(1-r)^{\varepsilon} \int_{\mathbf{B} \backslash B(0, r)} \Phi(\omega(1-r) f(y)) d y \leq C(1-r)^{\varepsilon}
\end{aligned}
$$

since $(\alpha+\varepsilon) p-\varepsilon-N+(N-1) / q>0$.

When $\varepsilon>0$ and $(\alpha+\varepsilon) p-\varepsilon-N \geq 0$, taking $0<\delta<(N-1) / q$, we have

$$
\begin{aligned}
& \Phi\left(\omega(1-|x|)(1-|x|)^{\varepsilon} I_{2}(x)\right) \\
& \leq C(1-|x|)^{\varepsilon} \int_{(1-|x|) / 2}^{2} t^{(\alpha+\varepsilon) p}\left(\frac{1}{|B(x, t)|} \int_{B(x, t)} \Phi\left(\omega(1-|x|) f_{2, x}(y)\right) d y\right) t^{-\varepsilon-1} d t \\
& \leq C(1-|x|)^{\varepsilon} \int_{\mathbf{B}}|x-y|^{-\delta} \Phi\left(\omega(1-|x|) f_{2, x}(y)\right) d y
\end{aligned}
$$

and

$$
\begin{aligned}
S_{q}\left(\Phi\left(\omega(1-r)(1-r)^{\varepsilon} I_{2}\right), r\right) & \leq C(1-r)^{\varepsilon} \int_{\mathbf{B}} S_{q}\left(|\cdot-y|^{-\delta} \chi_{E_{2, x}}(y), r\right) \Phi(\omega(1-r) f(y)) d y \\
& \leq C(1-r)^{\varepsilon} \int_{\mathbf{B} \backslash B(0, r)} \Phi(\omega(1-r) f(y)) d y \leq C(1-r)^{\varepsilon},
\end{aligned}
$$

which completes the proof of assertion (2). 
Lemma 2.7. Let $1 \leq q<\infty$.

(1) Suppose

$(\omega 4) \quad t^{\alpha p+\varepsilon_{0}-N+(N-1) / q} \omega(t)^{-p}$ is almost decreasing on $(0,1]$ for some $\varepsilon_{0}>0$. Let $0<\varepsilon<\varepsilon_{0} /(p-1)$. Then there exists a constant $C>0$ such that

$$
S_{q}\left(\Phi\left((1-r)^{\varepsilon} I_{3}\right), r\right) \leq C(1-r)^{(\alpha+\varepsilon) p-N+(N-1) / q} \omega(1-r)^{-p}
$$

for all $1 / 2<r<1$ and nonnegative measurable functions $f$ on $\mathbf{B}$ with $\|f\|_{M^{\Phi, \omega}(\mathbf{B})} \leq 1$.

(2) Suppose $\varepsilon>0$ and

$$
(N-1) / q>N-\alpha p-\varepsilon(p-1)
$$

Then there exists a constant $C>0$ such that

$$
S_{q}\left(\Phi\left((1-r)^{\varepsilon} I_{3}\right), r\right) \leq C(1-r)^{\varepsilon}
$$

for all $1 / 2<r<1$ and nonnegative measurable functions $f$ on $\mathbf{B}$ with $\|f\|_{M^{\Phi, \omega}(\mathbf{B})} \leq 1$.

Proof. Let $1 / 2<r=|x|<1$. First note from $(\omega 4)$ and $0<\varepsilon<\varepsilon_{0} /(p-1)$ that $t^{(\alpha+\varepsilon) p-\varepsilon-N+(N-1) / q} \omega(t)^{-p}$ is almost decreasing on $(0,1]$ and

$$
(\alpha+\varepsilon) p-\varepsilon-N+(N-1) / q<0 .
$$

Further, note that

$$
\int_{B(0,1 / 4)}|x-y|^{\alpha-N} f(y) d y \leq C \int_{B(0,1 / 4)} f(y) d y \leq C .
$$

As in the proof of Lemma 2.6, we have

$$
\begin{aligned}
I_{3}(x) & =\int_{\mathbf{B}}|x-y|^{\alpha-N} f_{3, x}(y) d y \\
& \leq C \int_{(1-|x|) / 2}^{2}\left(\frac{1}{|B(x, t)|} \int_{B(x, t)} f_{3, x}(y) d y\right) t^{\alpha-1} d t \\
& \leq C \int_{(1-|x|) / 2}^{2}\left(\frac{1}{|B(x, t)|} \int_{B(x, t)} t^{\alpha+\varepsilon} f_{3, x}(y) d y\right) t^{-\varepsilon-1} d t
\end{aligned}
$$

where $f_{3, x}(y)=f(y) \chi_{E_{3, x}}(y)$ with $E_{3, x}=\{y \in \mathbf{B} \backslash B(x,(1-|x|) / 2): 1-|y|>1-|x|\}$. Since $r \sim|y|$ for $y \in \mathbf{B} \backslash B(0,1 / 4)$, in the same way as in the proof of Lemma 2.6, we see from Lemma 2.2 that

$$
\begin{aligned}
S_{q}\left(\Phi\left((1-r)^{\varepsilon} I_{3}\right), r\right) & \leq C(1-r)^{\varepsilon}\left(\int_{\mathbf{B}} S_{q}\left(|\cdot-y|^{(\alpha+\varepsilon) p-\varepsilon-N} \chi_{E_{3, x}}(y), r\right) \Phi(f(y)) d y+1\right) \\
& \leq C(1-r)^{\varepsilon}\left(\int_{B(0, r)}(1-|y|)^{(\alpha+\varepsilon) p-\varepsilon-N+(N-1) / q} \Phi(f(y)) d y+1\right) .
\end{aligned}
$$


Let $j_{0}$ be the smallest integer such that $r \leq 1-2^{-j_{0}-1}$. Note here that

$$
\begin{aligned}
& \int_{B(0, r)}(1-|y|)^{(\alpha+\varepsilon) p-\varepsilon-N+(N-1) / q} \Phi(f(y)) d y \\
& \leq \sum_{j=0}^{j_{0}} \int_{A\left(0,1-2^{-j}\right)}(1-|y|)^{(\alpha+\varepsilon) p-\varepsilon-N+(N-1) / q} \Phi(f(y)) d y \\
& \leq C \sum_{j=0}^{j_{0}} 2^{-j((\alpha+\varepsilon) p-\varepsilon-N+(N-1) / q)} \int_{A\left(0,1-2^{-j}\right)} \Phi(f(y)) d y \\
& \leq C \sum_{j=0}^{j_{0}} 2^{-j((\alpha+\varepsilon) p-\varepsilon-N+(N-1) / q)} \omega\left(2^{-j}\right)^{-p} \\
& \leq C(1-r)^{(\alpha+\varepsilon) p-\varepsilon-N+(N-1) / q} \omega(1-r)^{-p}
\end{aligned}
$$

by $(\omega 4)$, which gives assertion (1).

For assertion (2), suppose $\varepsilon>0$ such that

$$
(N-1) / q>N-(\alpha+\varepsilon) p+\varepsilon>0 .
$$

Then, in the same way as in the proof of Lemma 2.6, we see from Lemma 2.2 that

$$
\begin{aligned}
& S_{q}\left(\Phi\left((1-r)^{\varepsilon} I_{3}\right), r\right) \\
& \leq C(1-r)^{\varepsilon}\left(\int_{\mathbf{B}} S_{q}\left(|\cdot-y|^{(\alpha+\varepsilon) p-\varepsilon-N} \chi_{E_{3, x}}(y), r\right) \Phi(f(y)) d y+1\right) \\
& \leq C(1-r)^{\varepsilon}\left(\int_{B(0, r)} \Phi(f(y)) d y+1\right) \leq C(1-r)^{\varepsilon} .
\end{aligned}
$$

When $\varepsilon>0$ and $(\alpha+\varepsilon) p-\varepsilon-N \geq 0$, we see that

$$
S_{q}\left(\Phi\left((1-r)^{\varepsilon} I_{3}\right), r\right) \leq C(1-r)^{\varepsilon}\left(\int_{B(0, r)} \Phi(f(y)) d y+1\right) \leq C(1-r)^{\varepsilon},
$$

as in the proof of Lemma 2.6. Thus the present lemma is proved.

Remark 2.8. If $\omega(r)=r^{-\nu}$, then $(\omega 4)$ holds in case $(N-1) / q<N-\alpha p-\nu p$.

\section{Spherical limits for Riesz potentials}

We are now ready to show our main result.

Theorem 3.1. Let $1 \leq q<\infty$.

(1) Suppose $(\omega 4)$ holds for some $\varepsilon_{0}>0$. If $0<\varepsilon<\min \left\{\alpha, \varepsilon_{0} /(p-1)\right\}$ and

$$
N-\alpha p+\varepsilon(p-1)-1<(N-1) / q<N-\alpha p-\varepsilon(p-1),
$$

then there exists a constant $C>0$ such that

$$
\liminf _{r \rightarrow 1-}(1-r)^{N-(\alpha+\varepsilon) p-(N-1) / q} \omega(1-r)^{p} S_{q}\left(\Phi\left((1-r)^{\varepsilon} I_{\alpha} f\right), r\right) \leq C
$$

for all nonnegative measurable functions $f$ with $\|f\|_{M^{\Phi, \omega}(\mathbf{B})} \leq 1$.

(2) If $0<\varepsilon<\alpha$ and

$$
\begin{aligned}
\max \{N-\alpha p-\varepsilon(p-1), N-\alpha p+\varepsilon(p-1)-1\} & <(N-1) / q \\
& <N-\alpha p+\varepsilon(p-1),
\end{aligned}
$$


then there exists a constant $C>0$ such that

$\liminf _{r \rightarrow 1-} \min \left\{(1-r)^{N-(\alpha+\varepsilon) p-(N-1) / q} \omega(1-r)^{p},(1-r)^{-\varepsilon}\right\} S_{q}\left(\Phi\left((1-r)^{\varepsilon} I_{\alpha} f\right), r\right) \leq C$

for all nonnegative measurable functions $f$ with $\|f\|_{M^{\Phi, \omega}(\mathbf{B})} \leq 1$.

(3) If $0<\varepsilon<\alpha$ and $(N-1) / q>N-\alpha p+\varepsilon(p-1)>0$, then there exists a constant $C>0$ such that

$$
\min \left\{(1-r)^{N-(\alpha+\varepsilon) p-(N-1) / q} \omega(1-r)^{p},(1-r)^{-\varepsilon}\right\} S_{q}\left(\Phi\left((1-r)^{\varepsilon} I_{\alpha} f\right), r\right) \leq C
$$

for all $1 / 2<r<1$ and all nonnegative measurable functions $f$ with $\|f\|_{M^{\Phi, \omega}(\mathbf{B})}$ $\leq 1$.

(4) If $0<\varepsilon<\alpha$ and $(\alpha-\varepsilon) p+\varepsilon-N>0$, then there exists a constant $C>0$ such that

$$
\min \left\{(1-r)^{N-(\alpha+\varepsilon) p} \omega(1-r)^{p},(1-r)^{-\varepsilon}\right\} S_{q}\left(\Phi\left((1-r)^{\varepsilon} I_{\alpha} f\right), r\right) \leq C
$$

for all $1 / 2<r<1$ and all nonnegative measurable functions $f$ with $\|f\|_{M^{\Phi, \omega}(\mathbf{B})}$ $\leq 1$.

Proof. We shall show assertion (1). Let $f$ be a nonnegative measurable function in $M^{\Phi, \omega}(\mathbf{B})$. For $x \in \mathbf{B}$, write

$$
I_{\alpha} f(x)=I_{1}(x)+I_{2}(x)+I_{3}(x)
$$

as before. Let $0<\varepsilon<\min \left\{\alpha, \varepsilon_{0} /(p-1)\right\}$ such that

$$
-1<(\alpha-\varepsilon) p+\varepsilon-N+(N-1) / q<(\alpha+\varepsilon) p-\varepsilon-N+(N-1) / q<0 .
$$

Set

$$
d=-(\alpha-\varepsilon) p-\varepsilon+N-(N-1) / q .
$$

Then $0<d<1$. First note by Lemma 2.6 (1) that

$$
(1-r)^{N-(\alpha+\varepsilon) p-(N-1) / q} S_{q}\left(\Phi\left(\omega(1-r)(1-r)^{\varepsilon} I_{2}\right), r\right) \leq C,
$$

so that by $(\Phi 4)$

$$
(1-r)^{N-(\alpha+\varepsilon) p-(N-1) / q} \omega(1-r)^{p} S_{q}\left(\Phi\left((1-r)^{\varepsilon} I_{2}\right), r\right) \leq C .
$$

By Lemma 2.7 (1), we have

$$
(1-r)^{N-(\alpha+\varepsilon) p-(N-1) / q} \omega(1-r)^{p} S_{q}\left(\Phi\left((1-r)^{\varepsilon} I_{3}\right), r\right) \leq C .
$$

Finally, we obtain by Lemma 2.4 (1)

$$
S_{q}\left(\Phi\left((1-r)^{-\varepsilon} \omega(1-r) I_{1}\right), r\right) \leq\left. C(1-r)^{-\varepsilon} \int_{A(0, r)}|r-| y\right|^{-d} g(y) d y,
$$

where $g(y)=\Phi(\omega(1-|y|) f(y))$. Therefore $(\Phi 4)$ gives

$$
\begin{aligned}
& (1-r)^{N-(\alpha+\varepsilon) p-(N-1) / q} \omega(1-r)^{p} S_{q}\left(\Phi\left((1-r)^{\varepsilon} I_{1}\right), r\right) \\
& \leq C(1-r)^{N-(\alpha+\varepsilon) p-(N-1) / q}(1-r)^{2 \varepsilon p} S_{q}\left(\Phi\left((1-r)^{-\varepsilon} \omega(1-r) I_{1}\right), r\right) \\
& \leq\left. C(1-r)^{d} \int_{A(0, r)}|r-| y\right|^{-d} g(y) d y .
\end{aligned}
$$

In view of Lemma 2.5, we can find a sequence $\left\{r_{j}\right\}$ of positive numbers such that $1-2^{-j+1}<r_{j}<1-2^{-j}$ and

$$
\sup _{j}\left(1-r_{j}\right)^{N-(\alpha+\varepsilon) p-(N-1) / q} \omega\left(1-r_{j}\right)^{p} S_{q}\left(\Phi\left(\left(1-r_{j}\right)^{\varepsilon} I_{1}\right), r_{j}\right) \leq C .
$$

Thus assertion (1) is obtained. 
Next we shall show assertion (2). Suppose $0<\varepsilon<\alpha$ such that

$$
N-\alpha p-\varepsilon(p-1)<(N-1) / q<N-\alpha p+\varepsilon(p-1) .
$$

By Lemmas 2.6 (2) and 2.7 (2), we obtain

$$
S_{q}\left(\Phi\left((1-r)^{\varepsilon} I_{2}\right), r\right) \leq C S_{q}\left(\Phi\left(\omega(1-r)(1-r)^{\varepsilon} I_{2}\right), r\right) \leq C(1-r)^{\varepsilon}
$$

and

$$
S_{q}\left(\Phi\left((1-r)^{\varepsilon} I_{3}\right), r\right) \leq C(1-r)^{\varepsilon}
$$

for all $1 / 2<r<1$. In view of $(\Phi 4)$, we obtain

$$
\begin{aligned}
& (1-r)^{N-(\alpha+\varepsilon) p-(N-1) / q} \omega(1-r)^{p} S_{q}\left(\Phi\left((1-r)^{\varepsilon} I_{1}\right), r\right) \\
& \leq C(1-r)^{N-(\alpha+\varepsilon) p-(N-1) / q}(1-r)^{2 p \varepsilon} S_{q}\left(\Phi\left(\omega(1-r)(1-r)^{-\varepsilon} I_{1}\right), r\right) \\
& \leq C(1-r)^{N-(\alpha-\varepsilon) p-(N-1) / q} S_{q}\left(\Phi\left(\omega(1-r)(1-r)^{-\varepsilon} I_{1}\right), r\right)
\end{aligned}
$$

for all $1 / 2<r<1$. Thus, by Lemma 2.4 (1), assertion (2) is proved.

For a proof of assertion (3), it suffices to apply Lemma 2.4 (2) in the proof of assertion (2).

For a proof of assertion (4), note that by our assumption

$$
(N-1) / q>0>N-\alpha p+\varepsilon(p-1)>N-\alpha p-\varepsilon(p-1)
$$

and by $(\Phi 4)$

$$
\begin{aligned}
& (1-r)^{N-(\alpha+\varepsilon) p} \omega(1-r)^{p} S_{q}\left(\Phi\left((1-r)^{\varepsilon} I_{1}\right), r\right) \\
& \leq C(1-r)^{N-(\alpha-\varepsilon) p} S_{q}\left(\Phi\left(\omega(1-r)(1-r)^{-\varepsilon} I_{1}\right), r\right)
\end{aligned}
$$

for all $1 / 2<r<1$. As in the proof of assertion (2), it suffices to apply Lemma 2.4 (3).

Remark 3.2. The first and third authors [17, Theorem 1] treated the existence of boundary limits for BLD functions $u$ on the unit ball $\mathbf{B}$ of $\mathbf{R}^{N}$ satisfying

$$
\int_{\mathbf{B}}|\nabla u(x)|^{p}(1-|x|)^{\gamma} d x<\infty
$$

where $\nabla$ denotes the gradient, $1<p<\infty$ and $-1<\gamma<p-1$. In fact, we showed that

$$
\liminf _{r \rightarrow 1-}(1-r)^{(N-p+\gamma) / p-(N-1) / q} S_{q}(u, r)=0
$$

when $q>0$ and $(N-p-1) /(p(N-1))<1 / q<(N-p+\gamma) /(p(N-1))$. If $u$ is in addition monotone in $\mathbf{B}$ in the sense of Lebesgue, then $u$ is shown to have weighted boundary limit zero (see [17, Theorem 2]).

When $\Phi(r)=r^{p}$ and $\omega(r)=r^{-\nu}$, we obtain the following corollary.

Corollary 3.3. Suppose $1 \leq q<\infty, \nu \geq 0$ and

$$
\frac{N-\alpha p-1}{N-1}<\frac{1}{q}<\frac{N-\alpha p-\nu p}{N-1} .
$$

Then

$$
\liminf _{r \rightarrow 1-}(1-r)^{(N-\alpha p-\nu p) / p-(N-1) /(p q)} S_{p q}\left(I_{\alpha} f, r\right)<\infty
$$

for all nonnegative measurable functions $f \in M^{p, \nu}(\mathbf{B})$. 
Proof. Let $f$ be a nonnegative measurable functions $f \in M^{p, \nu}(\mathbf{B})$. First note that $(\omega 4)$ holds for some $\varepsilon_{0}>0$. Take $0<\varepsilon<\min \left\{\alpha, \varepsilon_{0} /(p-1)\right\}$ such that

$$
N-\alpha p+\varepsilon(p-1)-1<(N-1) / q .
$$

Then Theorem 3.1 (1) gives

$$
\liminf _{r \rightarrow 1-}(1-r)^{(N-\alpha p-\nu p) / p-(N-1) /(p q)} S_{p q}\left(I_{\alpha} f, r\right)<\infty,
$$

as required.

Corollary 3.4. Suppose $1 \leq p \leq q<\infty$ and

$$
\frac{N-\alpha p-1}{p(N-1)}<\frac{1}{q}<\frac{N-\alpha p-\nu p}{p(N-1)} \text {. }
$$

Then

$$
\liminf _{r \rightarrow 1-}(1-r)^{(N-\alpha p-\nu p) / p-(N-1) / q} S_{q}\left(I_{\alpha} f, r\right)<\infty
$$

for all nonnegative measurable functions $f \in M^{p, \nu}(\mathbf{B})$.

Remark 3.5. We show that the exponent in Corollary 3.4 is the best possible. For this, let $1 \leq p \leq q<\infty$ and $\alpha+\nu-N / p+(N-1) / q<0$. Consider the function

$$
f(y)=|e-y|^{\nu-N / p}
$$

for $0<\nu<1 / p$, where $e=(0, \ldots, 0,1) \in \partial \mathbf{B}$. Then, by the proof of Lemma 2.2 and $0<\nu<1 / p$, we see that

$$
(1-r)^{-\nu p} \int_{\mathbf{B} \backslash B(0, r)}|f(y)|^{p} d y \leq C(1-r)^{-\nu p} \int_{r}^{1}(1-t)^{\nu p-1} d t \leq C
$$

for $1 / 2<r<1$. Moreover,

$$
\begin{aligned}
I_{\alpha} f(x) & \geq \int_{\mathbf{B} \cap B(x,|e-x| / 2)}|x-y|^{\alpha-N}|e-y|^{\nu-N / p} d y \\
& \geq C|e-x|^{\nu-N / p} \int_{\mathbf{B} \cap B(x,|e-x| / 2)}|x-y|^{\alpha-N} d y \\
& \geq C|e-x|^{\alpha+\nu-N / p}
\end{aligned}
$$

for $x \in \mathbf{B}$. Lemma 2.3 gives

$$
S_{q}\left(I_{\alpha} f, r\right) \geq C(1-r)^{\alpha+\nu-N / p+(N-1) / q}
$$

for $1 / 2<r<1$, since $\alpha+\nu-N / p+(N-1) / q<0$. Hence

$$
\liminf _{r \rightarrow 1-}(1-r)^{(N-\alpha p-\nu p) / p-(N-1) / q} S_{q}\left(I_{\alpha} f, r\right) \geq C>0 .
$$

Remark 3.6. Consider

$$
f(y)=\sum_{j=1}^{\infty}\left|y-\mathbf{e}_{j}\right|^{\nu-N / p} \chi_{B\left(0,\left(1-2^{-j}\right)+2^{-j-2}\right) \backslash B\left(0,\left(1-2^{-j}\right)-2^{-j-2}\right)}(y)
$$

for $0<\nu<1 / p$, where $\mathbf{e}=(0, \ldots, 0,1) \in \partial \mathbf{B}$ and $\mathbf{e}_{j}=\left(1-2^{-j}\right) \mathbf{e}$. Let $j_{0}$ be the largest integer such that $1-2^{-j_{0}}-2^{-j_{0}-2}<r \leq 1-2^{-j_{0}-1}-2^{-j_{0}-3}$. Then note from 
Lemma 2.2 that

$$
\begin{aligned}
\int_{\mathbf{B} \backslash B(0, r)} f(y)^{p} d y & \leq \sum_{j=j_{0}}^{\infty} \int_{B\left(0,\left(1-2^{-j}\right)+2^{-j-2}\right) \backslash B\left(0,\left(1-2^{-j}\right)-2^{-j-2}\right)}\left|y-\mathbf{e}_{j}\right|^{\nu p-N} d y \\
& \leq C \sum_{j=j_{0}}^{\infty} \int_{\left(1-2^{-j}\right)-2^{-j-2}}^{\left(1-2^{-j}\right)+2^{-j-2}}\left|t-\left(1-2^{-j}\right)\right|^{\nu p-1} d t \\
& \leq C \sum_{j=j_{0}}^{\infty} 2^{-j \nu p} \leq C(1-r)^{\nu p}
\end{aligned}
$$

since $0<\nu<1 / p$. Further,

$$
I_{\alpha} f(x) \geq C\left|x-\mathbf{e}_{j}\right|^{\alpha-N} \int_{B\left(\mathbf{e}_{j},\left|x-e_{j}\right| / 2\right)}\left|y-\mathbf{e}_{j}\right|^{\nu-N / p} d y \geq C\left|x-\mathbf{e}_{j}\right|^{\alpha+\nu-N / p}
$$

for $x \in B\left(\mathbf{e}_{j}, 2^{-j-2} / 2\right)$, since $\nu-N / p+N>0$. We see that

$$
S_{q}\left(I_{\alpha} f, 1-2^{-j}\right) \geq C\left(\int_{S\left(0,1-2^{-j}\right) \cap B\left(\mathbf{e}_{j}, 2^{-j-2} / 2\right)}\left|x-\mathbf{e}_{j}\right|^{(\alpha+\nu-N / p) q} d S(x)\right)^{1 / q}=\infty,
$$

when $(\alpha+\nu-N / p) q+N-1 \leq 0$. This implies the necessity of the lower limit in Theorem 3.1 when $\nu>0$ and

$$
\frac{N-1}{q} \leq \frac{N-\alpha p-\nu p}{p}
$$

Let $M_{0}^{\Phi, \omega}(\mathbf{B})$ denote the family of all measurable functions $f$ on $\mathbf{B}$ such that

$$
\lim _{r \rightarrow 1-} \int_{A(0, r)} \Phi(\omega(1-r)|f(y)|) d y=0 .
$$

With a slight modification of the proof of Theorem 3.1, we can prove the following result.

Corollary 3.7. Let $1 \leq q<\infty$. Suppose $(\omega 4)$ holds for some $\varepsilon_{0}>0$. If $0<\varepsilon<\min \left\{\alpha, \varepsilon_{0} /(p-1)\right\}$ and

$$
N-\alpha p+\varepsilon(p-1)-1<(N-1) / q<N-\alpha p-\varepsilon(p-1),
$$

then

$$
\liminf _{r \rightarrow 1-}(1-r)^{N-(\alpha+\varepsilon) p-(N-1) / q} \omega(1-r)^{p} S_{q}\left(\Phi\left((1-r)^{\varepsilon} I_{\alpha} f\right), r\right)=0
$$

for all nonnegative measurable functions $f \in M_{0}^{\Phi, \omega}(\mathbf{B})$.

Proof. Let $f$ be a nonnegative measurable function in $M_{0}^{\Phi, \omega}(\mathbf{B})$. For $x \in \mathbf{B}$, write

$$
I_{\alpha} f(x)=I_{1}(x)+I_{2}(x)+I_{3}(x)
$$

as before. Let $0<\varepsilon<\min \left\{\alpha, \varepsilon_{0} /(p-1)\right\}$ such that

$$
-1<(\alpha-\varepsilon) p+\varepsilon-N+(N-1) / q<(\alpha+\varepsilon) p-\varepsilon-N+(N-1) / q<0 .
$$

Set

$$
d=-(\alpha-\varepsilon) p-\varepsilon+N-(N-1) / q .
$$

Then $0<d<1$. First note by the proof of Lemma 2.6 (1) that

$$
(1-r)^{N-(\alpha+\varepsilon) p-(N-1) / q} S_{q}\left(\Phi\left(\omega(1-r)(1-r)^{\varepsilon} I_{2}\right), r\right) \leq C \int_{\mathbf{B} \backslash B(0, r)} \Phi(\omega(1-r) f(y)) d y,
$$


so that by $(\Phi 4)$

$$
(1-r)^{N-(\alpha+\varepsilon) p-(N-1) / q} \omega(1-r)^{p} S_{q}\left(\Phi\left((1-r)^{\varepsilon} I_{2}\right), r\right) \leq C \int_{\mathbf{B} \backslash B(0, r)} \Phi(\omega(1-r) f(y)) d y .
$$

Take $0<r_{0}<1$. We write

$$
\begin{aligned}
I_{3}(x)= & \int_{\left\{y \in \mathbf{B} \backslash B(x,(1-|x|) / 2): 1-|y|>1-|x|,|y| \leq r_{0}\right\}}|x-y|^{\alpha-N} f(y) d y \\
& +\int_{\left\{y \in \mathbf{B} \backslash B(x,(1-|x|) / 2): 1-|y|>1-|x|,|y|>r_{0}\right\}}|x-y|^{\alpha-N} f(y) d y \\
= & I_{3,1}(x)+I_{3,2}(x) .
\end{aligned}
$$

By $(\Phi 3)$ and the fact that $I_{3,1}(x) \leq C$, we have

$$
\begin{aligned}
& \liminf _{r \rightarrow 1-}(1-r)^{N-(\alpha+\varepsilon) p-(N-1) / q} \omega(1-r)^{p} S_{q}\left(\Phi\left((1-r)^{\varepsilon} I_{3,1}\right), r\right) \\
& \leq \liminf _{r \rightarrow 1-}(1-r)^{N-(\alpha+\varepsilon) p-(N-1) / q} \omega(1-r)^{p} S_{q}\left(\Phi\left((1-r)^{\varepsilon} C\right), r\right)=0
\end{aligned}
$$

and by the proof of Lemma $2.7(1)$

$$
\begin{aligned}
& (1-r)^{N-(\alpha+\varepsilon) p-(N-1) / q} \omega(1-r)^{p} S_{q}\left(\Phi\left((1-r)^{\varepsilon} I_{3,2}\right), r\right) \\
& \leq C \sup _{\left\{j \in \mathbf{N}: 1-2^{-j-1}>r_{0}\right\}} \omega\left(2^{-j}\right)^{p} \int_{A\left(0,1-2^{-j}\right)} \Phi(f(y)) d y
\end{aligned}
$$

for $r_{0}<r<1$, so that

$$
\begin{aligned}
& \liminf _{r \rightarrow 1-}(1-r)^{N-(\alpha+\varepsilon) p-(N-1) / q} \omega(1-r)^{p} S_{q}\left(\Phi\left((1-r)^{\varepsilon} I_{3}\right), r\right) \\
& \leq C \sup _{\left\{j \in \mathbf{N}: 1-2^{-j-1}>r_{0}\right\}} \omega\left(2^{-j}\right)^{p} \int_{A\left(0,1-2^{-j}\right)} \Phi(f(y)) d y .
\end{aligned}
$$

Letting $r_{0} \rightarrow 1$, we infer that

$$
\liminf _{r \rightarrow 1-}(1-r)^{N-(\alpha+\varepsilon) p-(N-1) / q} \omega(1-r)^{p} S_{q}\left(\Phi\left((1-r)^{\varepsilon} I_{3}\right), r\right)=0 .
$$

Finally, we obtain by Lemma 2.4

$$
S_{q}\left(\Phi\left((1-r)^{-\varepsilon} \omega(1-r) I_{1}\right), r\right) \leq\left. C(1-r)^{-\varepsilon} \int_{A(0, r)}|r-| y\right|^{-d} g(y) d y,
$$

where $g(y)=\Phi(\omega(1-|y|) f(y))$. Therefore $(\Phi 4)$ gives

$$
\begin{aligned}
& (1-r)^{N-(\alpha+\varepsilon) p-(N-1) / q} \omega(1-r)^{p} S_{q}\left(\Phi\left((1-r)^{\varepsilon} I_{1}\right), r\right) \\
& \leq C(1-r)^{N-(\alpha+\varepsilon) p-(N-1) / q}(1-r)^{2 \varepsilon p} S_{q}\left(\Phi\left((1-r)^{-\varepsilon} \omega(1-r) I_{1}\right), r\right) \\
& \leq\left. C(1-r)^{d} \int_{A(0, r)}|r-| y\right|^{-d} g(y) d y .
\end{aligned}
$$

In view of Lemma 2.5, we can find a sequence $\left\{r_{j}\right\}$ of positive numbers such that $r_{0}<1-2^{-j+1}<r_{j}<1-2^{-j}$ and

$$
\begin{aligned}
& \sup _{j}\left(1-r_{j}\right)^{N-(\alpha+\varepsilon) p-(N-1) / q} \omega\left(1-r_{j}\right)^{p} S_{q}\left(\Phi\left(\left(1-r_{j}\right)^{\varepsilon} I_{1}\right), r_{j}\right) \\
& \leq C \sup _{r_{0}<r<1} \int_{A(0, r)} \Phi(\omega(1-r) f(y)) d y .
\end{aligned}
$$

Thus, letting $r_{0} \rightarrow 1$, we obtain the required result. 


\section{Spherical limits for Riesz potentials II}

In this section we treat functions $f$ on $\mathbf{B}$ satisfying the weighted condition (1 $|y|)^{\beta p_{1}} f(y)^{p_{1}} \in M^{\Phi, \omega}(\mathbf{B})$ for $1<p_{1}<\infty$ and $\beta>0$.

Lemma 4.1. Let $1<p_{1}<\infty$ and $\beta>0$. Suppose $0<\alpha p_{1}-\alpha_{1}<\beta p_{1}<p_{1}-1$. Then there exists a constant $C>0$ such that

$$
\left((1-|x|)^{-\left(\alpha-\alpha_{1} / p_{1}\right)+\beta} I_{\alpha} f(x)\right)^{p_{1}} \leq C I_{\alpha_{1}} g(x)
$$

for all $x \in \mathbf{B} \backslash B(0,1 / 2)$ and nonnegative measurable functions $f \in L_{l o c}^{1}(\mathbf{B})$, where $g(y)=(1-|y|)^{\beta p_{1}} f(y)^{p_{1}}$.

Proof. Let $f$ be a nonnegative measurable function $f \in L_{l o c}^{1}(\mathbf{B})$. By Hölder's inequality and Lemma 2.2, we have

$$
\begin{aligned}
\int_{\mathbf{B}}|x-y|^{\alpha-N} f(y) d y \leq & \left(\int_{\mathbf{B}}|x-y|^{\left(\alpha-\alpha_{1} / p_{1}\right) p_{1}^{\prime}-N}(1-|y|)^{-\beta p_{1}^{\prime}} d y\right)^{1 / p_{1}^{\prime}} \\
& \times\left(\int_{\mathbf{B}}|x-y|^{\alpha_{1}-N}(1-|y|)^{\beta p_{1}} f(y)^{p_{1}} d y\right)^{1 / p_{1}} \\
\leq & \left(\int_{0}^{1}\left(\int_{S(0, r)}|x-y|^{\left(\alpha-\alpha_{1} / p_{1}\right) p_{1}^{\prime}-N} d S(y)\right)(1-r)^{-\beta p_{1}^{\prime}} d r\right)^{1 / p_{1}^{\prime}} \\
& \times\left(\int_{\mathbf{B}}|x-y|^{\alpha_{1}-N} g(y) d y\right)^{1 / p_{1}} \\
\leq & C\left(\int_{0}^{1}|| x|-r|^{\left(\alpha-\alpha_{1} / p_{1}\right) p_{1}^{\prime}-1}(1-r)^{-\beta p_{1}^{\prime}} d r\right)^{1 / p_{1}^{\prime}} \\
& \times\left(\int_{\mathbf{B}}|x-y|^{\alpha_{1}-N} g(y) d y\right)^{1 / p_{1}},
\end{aligned}
$$

where $g(y)=(1-|y|)^{\beta p_{1}} f(y)^{p_{1}}$. Since $\left(\alpha-\alpha_{1} / p_{1}\right) p_{1}^{\prime}>0,-\beta p_{1}^{\prime}+1>0$ and $\left(\alpha-\alpha_{1} / p_{1}\right) p_{1}^{\prime}-\beta p_{1}^{\prime}+1<1$ by our assumptions, the Riesz composition formula (see e.g. $[15$, p. 59]) yields

$$
\int_{\mathbf{B}}|x-y|^{\alpha-N} f(y) d y \leq C(1-|x|)^{\left(\alpha-\alpha_{1} / p_{1}\right)-\beta}\left(\int_{\mathbf{B}}|x-y|^{\alpha_{1}-N} g(y) d y\right)^{1 / p_{1}},
$$

as required.

In view of Lemma 4.1 and Theorem 3.1, we obtain the following theorem.

Theorem 4.2. Let $1 \leq q<\infty$ and let $0<\alpha p_{1}-\alpha_{1}<\beta p_{1}<p_{1}-1$.

(1) Suppose $(\omega 4)$ holds for some $\varepsilon_{0}>0$ and $\alpha$ replaced by $\alpha_{1}$. If $0<\varepsilon<$ $\min \left\{\alpha_{1}, \varepsilon_{0} /(p-1)\right\}$ and

$$
N-\alpha_{1} p+\varepsilon(p-1)-1<(N-1) / q<N-\alpha_{1} p-\varepsilon(p-1),
$$

then there exists a constant $C>0$ such that

$$
\begin{aligned}
& \liminf _{r \rightarrow 1-}(1-r)^{N-\left(\alpha_{1}+\varepsilon\right) p-(N-1) / q} \omega(1-r)^{p} \\
& \times S_{q}\left(\Phi\left((1-r)^{\varepsilon}\left((1-r)^{-\left(\alpha-\alpha_{1} / p_{1}\right)+\beta} I_{\alpha} f\right)^{p_{1}}\right), r\right) \leq C
\end{aligned}
$$

for all nonnegative measurable functions $f$ with $\|g\|_{M^{\Phi, \omega}(\mathbf{B})} \leq 1$, where $g(y)=$ $(1-|y|)^{\beta p_{1}} f(y)^{p_{1}}$. 
(2) If $0<\varepsilon<\alpha_{1}$ and

$$
\begin{aligned}
\max \left\{N-\alpha_{1} p-\varepsilon(p-1), N-\alpha_{1} p+\varepsilon(p-1)-1\right\} & <(N-1) / q \\
& <N-\alpha_{1} p+\varepsilon(p-1),
\end{aligned}
$$

then there exists a constant $C>0$ such that

$$
\begin{aligned}
& \liminf _{r \rightarrow 1-} \min \left\{(1-r)^{N-\left(\alpha_{1}+\varepsilon\right) p-(N-1) / q} \omega(1-r)^{p},(1-r)^{-\varepsilon}\right\} \\
& \times S_{q}\left(\Phi\left((1-r)^{\varepsilon}\left((1-r)^{-\left(\alpha-\alpha_{1} / p_{1}\right)+\beta} I_{\alpha} f\right)^{p_{1}}\right), r\right) \leq C
\end{aligned}
$$

for all nonnegative measurable functions $f$ with $\|g\|_{M^{\Phi, \omega}(\mathbf{B})} \leq 1$, where $g(y)=$ $(1-|y|)^{\beta p_{1}} f(y)^{p_{1}}$.

(3) If $0<\varepsilon<\alpha_{1}$ and $(N-1) / q>N-\alpha_{1} p+\varepsilon(p-1)>0$, then there exists a constant $C>0$ such that

$$
\begin{aligned}
& \min \left\{(1-r)^{N-\left(\alpha_{1}+\varepsilon\right) p-(N-1) / q} \omega(1-r)^{p},(1-r)^{-\varepsilon}\right\} \\
& \times S_{q}\left(\Phi\left((1-r)^{\varepsilon}\left((1-r)^{-\left(\alpha-\alpha_{1} / p_{1}\right)+\beta} I_{\alpha} f\right)^{p_{1}}\right), r\right) \leq C
\end{aligned}
$$

for all $1 / 2<r<1$ and all nonnegative measurable functions $f$ with $\|g\|_{M^{\Phi, \omega}(\mathbf{B})}$ $\leq 1$, where $g(y)=(1-|y|)^{\beta p_{1}} f(y)^{p_{1}}$.

(4) If $0<\varepsilon<\alpha_{1}$ and $\left(\alpha_{1}-\varepsilon\right) p+\varepsilon-N>0$, then there exists a constant $C>0$ such that

$\min \left\{(1-r)^{N-\left(\alpha_{1}+\varepsilon\right) p} \omega(1-r)^{p},(1-r)^{-\varepsilon}\right\} S_{q}\left(\Phi\left((1-r)^{\varepsilon}\left((1-r)^{-\left(\alpha-\alpha_{1} / p_{1}\right)+\beta} I_{\alpha} f\right)^{p_{1}}\right), r\right) \leq C$

for all $1 / 2<r<1$ and all nonnegative measurable functions $f$ with $\|g\|_{M^{\Phi, \omega}(\mathbf{B})}$ $\leq 1$, where $g(y)=(1-|y|)^{\beta p_{1}} f(y)^{p_{1}}$.

When $\Phi(r)=r^{p}$ and $\omega(r)=1$, we obtain the following corollary.

Corollary 4.3. If $1 \leq q<\infty, 0<\alpha p_{1}-\alpha_{1}<\beta p_{1}<p_{1}-1$ and

$$
\frac{N-\alpha_{1} p-1}{N-1}<\frac{1}{q}<\frac{N-\alpha_{1} p}{N-1}
$$

then

$$
\liminf _{r \rightarrow 1-}(1-r)^{N-(\alpha-\beta) p p_{1}-(N-1) / q} S_{q}\left(\left(I_{\alpha} f\right)^{p p_{1}}, r\right)<\infty
$$

for all nonnegative measurable functions $f$ such that

$$
\int_{\mathbf{B}} f(y)^{p p_{1}}(1-|y|)^{\beta p_{1} p} d y<\infty
$$

\section{Green potentials}

Let $G(x, y)$ be a Green kernel on $\mathbf{B}$. When $N \geq 3$, there exists a constant $C>0$ such that

$$
C^{-1} \frac{(1-|x|)(1-|y|)}{|x-y|^{N-2}\left|x^{*}-y\right|^{2}} \leq G(x, y) \leq C \frac{(1-|x|)(1-|y|)}{|x-y|^{N-2}\left|x^{*}-y\right|^{2}} \leq C \frac{(1-|x|)(1-|y|)}{|x-y|^{N}}
$$

for $x, y \in \mathbf{B}$, where $x^{*}$ is the inversion of $x$ with respect to $S(0,1)$. 
For $f \in L_{\text {loc }}^{1}(\mathbf{B})$ and $x \in \mathbf{B}$, we write

$$
\begin{aligned}
G f(x)= & \int_{\mathbf{B}} G(x, y) f(y) d y=\int_{B(x,(1-|x|) / 2)} G(x, y) f(y) d y \\
& +\int_{\{y \in \mathbf{B} \backslash B(x,(1-|x|) / 2): 1-|y| \leq 1-|x|\}} G(x, y) f(y) d y \\
& +\int_{\{y \in \mathbf{B} \backslash B(x,(1-|x|) / 2): 1-|y|>1-|x|\}} G(x, y) f(y) d y \\
= & G_{1}(x)+G_{2}(x)+G_{3}(x) .
\end{aligned}
$$

Lemma 5.1. Let $1 \leq q<\infty$.

(1) Suppose $\varepsilon>0$ and

$$
(N-1) / q<N-\varepsilon(p-1) .
$$

Then there exists a constant $C>0$ such that

$$
S_{q}\left(\Phi\left((1-r)^{-1+\varepsilon} G_{2}\right), r\right) \leq C(1-r)^{\varepsilon p-N+(N-1) / q} \omega(1-r)^{-p}
$$

for all $1 / 2<r<1$ and nonnegative measurable functions $f$ on $\mathbf{B}$ with $\|F\|_{M^{\Phi, \omega}(\mathbf{B})} \leq 1$, where $F(y)=(1-|y|) f(y)$.

(2) Suppose $\varepsilon>0$ and

$$
(N-1) / q>N-\varepsilon(p-1) .
$$

Then there exists a constant $C>0$ such that

$$
S_{q}\left(\Phi\left((1-r)^{-1+\varepsilon} G_{2}\right), r\right) \leq C(1-r)^{\varepsilon} \omega(1-r)^{-p}
$$

for all $1 / 2<r<1$ and nonnegative measurable functions $f$ on $\mathbf{B}$ with $\|F\|_{M^{\Phi, \omega}(\mathbf{B})} \leq 1$, where $F(y)=(1-|y|) f(y)$.

Proof. Let $\varepsilon>0$ such that

$$
\varepsilon(p-1)-N+(N-1) / q<0 .
$$

For $1 / 2<r=|x|<1$, we have

$$
\begin{aligned}
G_{2}(x) & \leq C \int_{\mathbf{B}}(1-|x|)(1-|y|)|x-y|^{-N} f_{2, x}(y) d y \\
& \leq C(1-|x|) \int_{(1-|x|) / 2}^{2}\left(\frac{1}{|B(x, t)|} \int_{B(x, t)}(1-|y|) f_{2, x}(y) d y\right) t^{-1} d t \\
& \leq C(1-|x|) \int_{(1-|x|) / 2}^{2}\left(\frac{1}{|B(x, t)|} \int_{B(x, t)} t^{\varepsilon}(1-|y|) f_{x, 2}(y) d y\right) t^{-\varepsilon-1} d t,
\end{aligned}
$$

where $f_{2, x}(y)=f(y) \chi_{E_{2, x}}(y)$ with $E_{2, x}=\{y \in \mathbf{B} \backslash B(x,(1-|x|) / 2): 1-|y| \leq 1-|x|\}$.

We have by Jensen's inequality and $(\Phi 3)$

$$
\begin{aligned}
& \Phi\left((1-|x|)^{-1+\varepsilon} G_{2}(x)\right) \\
& \leq C(1-|x|)^{\varepsilon} \int_{(1-|x|) / 2}^{2}\left(\frac{1}{|B(x, t)|} \int_{B(x, t)} \Phi\left(t^{\varepsilon}(1-|y|) f_{2, x}(y)\right) d y\right) t^{-\varepsilon-1} d t \\
& \leq C(1-|x|)^{\varepsilon} \int_{(1-|x|) / 2}^{2} t^{\varepsilon p}\left(\frac{1}{|B(x, t)|} \int_{B(x, t)} \Phi\left((1-|y|) f_{2, x}(y)\right) d y\right) t^{-\varepsilon-1} d t \\
& \leq C(1-|x|)^{\varepsilon} \int_{\mathbf{B}}|x-y|^{\varepsilon(p-1)-N} \Phi\left((1-|y|) f_{2, x}(y)\right) d y .
\end{aligned}
$$


Hence Minkowski's inequality, Lemma 2.2 and (\$3) yield

$$
\begin{aligned}
& S_{q}\left(\Phi\left((1-r)^{-1+\varepsilon} G_{2}\right), r\right) \\
& \leq C(1-r)^{\varepsilon} \int_{\mathbf{B}} S_{q}\left(|\cdot-y|^{\varepsilon(p-1)-N} \chi_{E_{2, x}}(y), r\right) \Phi\left((1-|y|) f_{2, x}(y)\right) d y \\
& \leq C(1-r)^{\varepsilon}(1-r)^{\varepsilon(p-1)-N+(N-1) / q} \int_{\mathbf{B} \backslash B(0, r)} \Phi(F(y)) d y \\
& \leq C(1-r)^{\varepsilon p-N+(N-1) / q} \omega(1-r)^{-p}
\end{aligned}
$$

since $\varepsilon(p-1)-N+(N-1) / q<0$, which gives assertion (1).

To show assertion (2), suppose $\varepsilon>0$ such that $\varepsilon(p-1)-N<0$ and $\varepsilon(p-1)-$ $N+(N-1) / q>0$. Then we have by Lemma 2.2

$$
\begin{aligned}
& S_{q}\left(\Phi\left((1-r)^{-1+\varepsilon} G_{2}\right), r\right) \\
& \leq C(1-r)^{\varepsilon} \int_{\mathbf{B}} S_{q}\left(|\cdot-y|^{\varepsilon(p-1)-N} \chi_{E_{2, x}}(y), r\right) \Phi\left((1-|y|) f_{2, x}(y)\right) d y \\
& \leq C(1-r)^{\varepsilon} \int_{\mathbf{B} \backslash B(0, r)} \Phi(F(y)) d y \leq C(1-r)^{\varepsilon} \omega(1-r)^{-p}
\end{aligned}
$$

since $\varepsilon(p-1)-N+(N-1) / q>0$.

When $\varepsilon>0, \varepsilon(p-1)-N \geq 0$ and $\varepsilon(p-1)-N+(N-1) / q>0$, taking $0<\delta<(N-1) / q$, we have

$$
\begin{aligned}
& \Phi\left((1-|x|)^{-1+\varepsilon} G_{2}(x)\right) \\
& \leq C(1-|x|)^{\varepsilon} \int_{(1-|x|) / 2}^{2} t^{\varepsilon p}\left(\frac{1}{|B(x, t)|} \int_{B(x, t)} \Phi\left((1-|y|) f_{2, x}(y)\right) d y\right) t^{-\varepsilon-1} d t \\
& \leq C(1-|x|)^{\varepsilon} \int_{\mathbf{B}}|x-y|^{-\delta} \Phi\left((1-|y|) f_{2, x}(y)\right) d y
\end{aligned}
$$

and

$$
\begin{aligned}
S_{q}\left(\Phi\left((1-r)^{-1+\varepsilon} G_{2}\right), r\right) & \leq C(1-r)^{\varepsilon} \int_{\mathbf{B}} S_{q}\left(|\cdot-y|^{-\delta} \chi_{E_{2, x}}(y), r\right) \Phi(F(y)) d y \\
& \leq C(1-r)^{\varepsilon} \int_{\mathbf{B} \backslash B(0, r)} \Phi(F(y)) d y \leq C(1-r)^{\varepsilon} \omega(1-r)^{-p},
\end{aligned}
$$

which completes the proof of assertion (2).

Lemma 5.2. Let $1 \leq q<\infty$.

(1) Suppose

( $\omega 5) \quad t^{\varepsilon_{0}-N+(N-1) / q} \omega(t)^{-p}$ is almost decreasing on $(0,1)$ for some $\varepsilon_{0}>0$.

Let $0<\varepsilon<\varepsilon_{0} /(p-1)$. Then there exists a constant $C>0$ such that

$$
S_{q}\left(\Phi\left((1-r)^{-1+\varepsilon} G_{3}\right), r\right) \leq C(1-r)^{\varepsilon p-N+(N-1) / q} \omega(1-r)^{-p}
$$

for all $1 / 2<r<1$ and nonnegative measurable functions $f$ on $\mathbf{B}$ with $\|F\|_{M^{\Phi, \omega}(\mathbf{B})} \leq 1$, where $F(y)=(1-|y|) f(y)$.

(2) Suppose $\varepsilon>0$ and

$$
(N-1) / q>N-\varepsilon(p-1) .
$$

Then there exists a constant $C>0$ such that

$$
S_{q}\left(\Phi\left((1-r)^{-1+\varepsilon} G_{3}\right), r\right) \leq C(1-r)^{\varepsilon}
$$


for all $1 / 2<r<1$ and nonnegative measurable functions $f$ on $\mathbf{B}$ with $\|F\|_{M^{\Phi, \omega}(\mathbf{B})} \leq 1$, where $F(y)=(1-|y|) f(y)$.

Proof. First note from $(\omega 5)$ and $0<\varepsilon<\varepsilon_{0} /(p-1)$ that $t^{\varepsilon(p-1)-N+(N-1) / q} \omega(t)^{-p}$ is almost decreasing on $(0,1)$ and

$$
\varepsilon(p-1)-N+(N-1) / q<0 .
$$

In the same way as above, we obtain

$$
S_{q}\left(\Phi\left((1-r)^{-1+\varepsilon} G_{3}\right), r\right) \leq C(1-r)^{\varepsilon}\left(\int_{B(0, r)}(1-|y|)^{\varepsilon(p-1)-N+(N-1) / q} \Phi(F(y)) d y+1\right) .
$$

Let $j_{0}$ be the smallest integer such that $r \leq 1-2^{-j_{0}-1}$. Note here that

$$
\begin{aligned}
& \int_{B(0, r)}(1-|y|)^{\varepsilon(p-1)-N+(N-1) / q} \Phi(F(y)) d y \\
& \leq \sum_{j=0}^{j_{0}} \int_{A\left(0,1-2^{-j}\right)}(1-|y|)^{\varepsilon(p-1)-N+(N-1) / q} \Phi(F(y)) d y \\
& \leq C \sum_{j=0}^{j_{0}} 2^{-j(\varepsilon(p-1)-N+(N-1) / q)} \int_{A\left(0,1-2^{-j}\right)} \Phi(F(y)) d y \\
& \leq C \sum_{j=0}^{j_{0}} 2^{-j(\varepsilon(p-1)-N+(N-1) / q)} \omega\left(2^{-j}\right)^{-p} \\
& \leq C(1-r)^{\varepsilon(p-1)-N+(N-1) / q} \omega(1-r)^{-p}
\end{aligned}
$$

by ( $\omega 5)$, which gives assertion (1).

Assertion (2) is proved as in the proof of Lemma 2.7 (2).

Theorem 5.3. Let $1 \leq q<\infty$.

(1) Suppose ( $\omega 5)$ holds for some $\varepsilon_{0}>0$. If $0<\varepsilon<\min \left\{1, \varepsilon_{0} /(p-1)\right\}$ and

$$
N-2 p-1+\varepsilon(p-1)<(N-1) / q<N-2 p-\varepsilon(p-1),
$$

then there exists a constant $C>0$ such that

$$
\liminf _{r \rightarrow 1-}(1-r)^{N-2 p-(N-1) / q+\varepsilon p} \omega(1-r)^{p} S_{q}\left(\Phi\left((1-r)^{1-\varepsilon} G f\right), r\right) \leq C
$$

for all nonnegative measurable functions $f$ with $\|F\|_{M^{\Phi, \omega}(\mathbf{B})} \leq 1$, where $F(y)=(1-|y|) f(y)$.

(2) Suppose $(\omega 5)$ holds for some $\varepsilon_{0}>0$. If $0<\varepsilon<\min \left\{1, \varepsilon_{0} /(p-1)\right\}, 2 p-N-$ $\varepsilon(p-1)<0$ and

$$
N-2 p-\varepsilon(p-1)<(N-1) / q<N-\varepsilon(p-1),
$$

then there exists a constant $C>0$ such that

$$
(1-r)^{N-2 p-(N-1) / q+\varepsilon p} \omega(1-r)^{p} S_{q}\left(\Phi\left((1-r)^{1-\varepsilon} G f\right), r\right) \leq C
$$

for all $1 / 2<r<1$ and nonnegative measurable functions $f$ with $\|F\|_{M^{\Phi, \omega}(\mathbf{B})} \leq$ 1 , where $F(y)=(1-|y|) f(y)$.

(3) If $0<\varepsilon<1,2 p-N<\varepsilon(p-1)$ and $(N-1) / q>N-\varepsilon(p-1)$, then there exists a constant $C>0$ such that

$$
(1-r)^{-2(1-\varepsilon) p-\varepsilon} S_{q}\left(\Phi\left((1-r)^{1-\varepsilon} G f\right), r\right) \leq C
$$


for all $1 / 2<r<1$ and all nonnegative measurable functions $f$ with $\|F\|_{M^{\Phi, \omega}(\mathbf{B})}$ $\leq 1$, where $F(y)=(1-|y|) f(y)$. write

Proof. Let $f$ be a nonnegative measurable function in $M^{\Phi, \omega}(\mathbf{B})$. For $x \in \mathbf{B}$, as before.

$$
G f(x)=G_{1}(x)+G_{2}(x)+G_{3}(x)
$$

Let $0<\varepsilon<\min \left\{1, \varepsilon_{0} /(p-1)\right\}$ such that

$$
-1<(2-\varepsilon) p+\varepsilon-N+(N-1) / q<(2+\varepsilon) p-\varepsilon-N+(N-1) / q<0 .
$$

Set

$$
d=\varepsilon(p-1)-(N-1) / q+N-2 p .
$$

Then $0<d<1$. Since $\varepsilon(p-1)-N+(N-1) / q<\varepsilon_{0}-N+(N-1) / q \leq 0$ by $(\omega 5)$, one notes by Lemmas $5.1(1), 5.2(1)$ and $(\Phi 3)$ that

$$
\begin{aligned}
& (1-r)^{N-2 p+\varepsilon p-(N-1) / q} \omega(1-r)^{p} S_{q}\left(\Phi\left((1-r)^{1-\varepsilon} G_{2}\right), r\right) \\
& \leq C(1-r)^{N-2 p+\varepsilon p-(N-1) / q} \omega(1-r)^{p}(1-r)^{2(1-\varepsilon) p} S_{q}\left(\Phi\left((1-r)^{-1+\varepsilon} G_{2}\right), r\right) \\
& \leq C(1-r)^{N-\varepsilon p-(N-1) / q} \omega(1-r)^{p} S_{q}\left(\Phi\left((1-r)^{-1+\varepsilon} G_{2}\right), r\right) \leq C
\end{aligned}
$$

and

$$
(1-r)^{N-2 p+\varepsilon p-(N-1) / q} \omega(1-r)^{p} S_{q}\left(\Phi\left((1-r)^{1-\varepsilon} G_{3}\right), r\right) \leq C .
$$

Next we see that $G_{1}(x) \leq \int_{B(x,(1-|x|) / 2)}|x-y|^{2-N} f(y) d y$, and hence

$$
(1-|x|) G_{1}(x) \leq C \int_{B(x,(1-|x|) / 2)}|x-y|^{2-N}(1-|y|) f(y) d y .
$$

By Lemma 2.4 (1) with $\alpha=2$, we obtain

$$
S_{q}\left(\Phi\left(\omega(1-r)(1-r)^{1-\varepsilon} G_{1}\right), r\right) \leq C(1-r)^{-\varepsilon} \int_{A(0, r)}|r-| y||^{-d} g(y) d y,
$$

where $g(y)=\Phi(\omega(1-|y|) F(y))$ with $F(y)=(1-|y|) f(y)$. Therefore we establish by $(\Phi 4)$

$$
\begin{aligned}
& (1-r)^{N-2 p+\varepsilon p-(N-1) / q} \omega(1-r)^{p} S_{q}\left(\Phi\left((1-r)^{1-\varepsilon} G_{1}\right), r\right) \\
& \leq C(1-r)^{N-2 p+\varepsilon p-(N-1) / q} S_{q}\left(\Phi\left(\omega(1-r)(1-r)^{1-\varepsilon} G_{1}\right), r\right) \\
& \leq\left. C t^{d} \int_{A(0, r)}|r-| y\right|^{-d} g(y) d y .
\end{aligned}
$$

In view of Lemma 2.5, we can find a sequence $\left\{r_{j}\right\}$ of positive numbers such that $1-2^{-j+1}<r_{j}<1-2^{-j}$ and

$$
\sup _{j}\left(1-r_{j}\right)^{N-2 p+\varepsilon p-(N-1) / q} \omega\left(1-r_{j}\right)^{p} S_{q}\left(\Phi\left(\left(1-r_{j}\right)^{1-\varepsilon} G_{1}\right), r_{j}\right) \leq C,
$$

which proves assertion (1). and

To show assertion $(2)$, suppose $0<\varepsilon<\min \left\{1, \varepsilon_{0} /(p-1)\right\}, 2 p-N-\varepsilon(p-1)<0$

$$
\varepsilon(p-1)+N-2 p<(N-1) / q<N-\varepsilon(p-1) .
$$

Then, for $1 / 2<r<1$, we see from Lemmas 5.1 (1), 5.2 (1) and (\$3) that

$$
(1-r)^{N-2 p+\varepsilon p-(N-1) / q} \omega(1-r)^{p} S_{q}\left(\Phi\left((1-r)^{1-\varepsilon} G_{2}\right), r\right) \leq C
$$


and

$$
(1-r)^{N-2 p+\varepsilon p-(N-1) / q} \omega(1-r)^{p} S_{q}\left(\Phi\left((1-r)^{1-\varepsilon} G_{3}\right), r\right) \leq C,
$$

as above. By Lemma 2.4 (2) with $\alpha=2$, we obtain

$$
\begin{aligned}
& (1-r)^{N-2 p+\varepsilon p-(N-1) / q} \omega(1-r)^{p} S_{q}\left(\Phi\left((1-r)^{1-\varepsilon} G_{1}\right), r\right) \\
& \leq C(1-r)^{N-2 p+\varepsilon p-(N-1) / q} S_{q}\left(\Phi\left(\omega(1-r)(1-r)^{1-\varepsilon} G_{1}\right), r\right) \leq C,
\end{aligned}
$$

which proves assertion (2).

For a proof of (3), suppose $0<\varepsilon<1,2 p-N<\varepsilon(p-1)$ and $(N-1) / q>$ $N-\varepsilon(p-1)$. Then Lemmas $5.1(2), 5.2(2)$ yield

$$
\begin{aligned}
& (1-r)^{-2(1-\varepsilon) p-\varepsilon} S_{q}\left(\Phi\left((1-r)^{1-\varepsilon} G_{2}\right), r\right) \\
& \leq C(1-r)^{-2(1-\varepsilon) p-\varepsilon} \omega(1-r)^{p}(1-r)^{2(1-\varepsilon) p} S_{q}\left(\Phi\left((1-r)^{-1+\varepsilon} G_{2}\right), r\right) \\
& \leq C(1-r)^{-\varepsilon} \omega(1-r)^{p} S_{q}\left(\Phi\left((1-r)^{-1+\varepsilon} G_{2}\right), r\right) \leq C
\end{aligned}
$$

and

$$
(1-r)^{-2(1-\varepsilon) p-\varepsilon} S_{q}\left(\Phi\left((1-r)^{1-\varepsilon} G_{3}\right), r\right) \leq C
$$

for all $1 / 2<r<1$. Further we see from Lemma 2.4 (2) with $\alpha=2$ that

$$
\begin{aligned}
& (1-r)^{-2(1-\varepsilon) p-\varepsilon} S_{q}\left(\Phi\left((1-r)^{1-\varepsilon} G_{1}\right), r\right) \\
& \leq C(1-r)^{-2(1-\varepsilon) p-\varepsilon} S_{q}\left(\Phi\left((1-r)^{1-\varepsilon} \omega(1-r) G_{1}\right), r\right) \\
& \leq C(1-r)^{\varepsilon(p-1)-N+(N-1) / q} \leq C
\end{aligned}
$$

since

$$
(N-1) / q>N-\varepsilon(p-1)>N-2 p+\varepsilon(p-1)
$$

by $\varepsilon<1<p /(p-1)$. Hence we obtain assertion (3).

We can prove the following result in the same way as Corollary 3.7.

Theorem 5.4. Let $1 \leq q<\infty$ and $f$ be a nonnegative measurable function such that $F \in M_{0}^{\Phi, \omega}(\mathbf{B})$, where $F(y)=(1-|y|) f(y)$.

(1) Suppose $(\omega 5)$ holds for some $\varepsilon_{0}>0$. If $0<\varepsilon<\min \left\{1, \varepsilon_{0} /(p-1)\right\}$ and

$$
N-2 p-1+\varepsilon(p-1)<(N-1) / q<N-2 p-\varepsilon(p-1),
$$

then

$$
\liminf _{r \rightarrow 1-}(1-r)^{N-2 p-(N-1) / q+\varepsilon p} \omega(1-r)^{p} S_{q}\left(\Phi\left((1-r)^{1-\varepsilon} G f\right), r\right)=0 .
$$

(2) Suppose ( $\omega 5)$ holds for some $\varepsilon_{0}>0$. If $0<\varepsilon<\min \left\{1, \varepsilon_{0} /(p-1)\right\}, 2 p-N-$ $\varepsilon(p-1)<0$ and

$$
N-2 p-\varepsilon(p-1)<(N-1) / q<N-\varepsilon(p-1),
$$

then

$$
\lim _{r \rightarrow 1-}(1-r)^{N-2 p-(N-1) / q+\varepsilon p} \omega(1-r)^{p} S_{q}\left(\Phi\left((1-r)^{1-\varepsilon} G f\right), r\right)=0 .
$$

(3) If $0<\varepsilon<1,2 p-N<\varepsilon(p-1)$ and $(N-1) / q>N-\varepsilon(p-1)$, then

$$
\lim _{r \rightarrow 1-}(1-r)^{-2(1-\varepsilon) p-\varepsilon} S_{q}\left(\Phi\left((1-r)^{1-\varepsilon} G f\right), r\right)=0 .
$$

Remark 5.5. Gardiner [4] proved that for a Green potential $G \mu$ in B

(1) when $(N-1) /(N-2) \leq q<(N-1) /(N-3)$,

$$
\liminf _{r \rightarrow 1-}(1-r)^{N-1-(N-1) / q} S_{q}(G \mu, r)=0 ;
$$


(2) when $1 \leq q<(N-1) /(N-2)$,

$$
\lim _{r \rightarrow 1-}(1-r)^{N-1-(N-1) / q} S_{q}(G \mu, r)=0 .
$$

To obtain this result, we need modify Theorem 5.3 as in Corollary 3.7.

\section{Monotone functions}

A continuous function $u$ is said to be monotone in $\Omega$ in the sense of Lebesgue [7], if for every relatively compact subdomain $G$ of $\Omega$ we have

$$
\max _{\bar{G}} u=\max _{\partial G} u \text { and } \min _{\bar{G}} u=\min _{\partial G} u .
$$

For monotone functions, see Koskela-Manfredi-Villamor [6], Manfredi-Villamor [9, 10], the first author [14, 15], Villamor-Li [24] and Vuorinen [25, 26].

Theorem 6.1. Let $p_{1}>N-1$ and $p_{1} \leq q<\infty$. Suppose

( $\omega 6) t^{\varepsilon_{0}-\left(N-p_{1}-1\right) / p_{1}+(N-1) / q} \Phi^{-1}\left(t^{-1} \omega(t)^{-p}\right)^{1 / p_{1}}$ is almost decreasing in $(0,1)$ for some $\varepsilon_{0}>0$.

Then there exists a constant $C>0$ such that

$$
\limsup _{r \rightarrow 1-}(1-r)^{\left(N-p_{1}-1\right) / p_{1}-(N-1) / q} \Phi^{-1}\left((1-r)^{-1} \omega(1-r)^{-p}\right)^{-1 / p_{1}} S_{q}(u, r) \leq C
$$

for all monotone functions $u$ on $\mathbf{B}$ such that $\|h\|_{M^{\Phi, \omega}(\mathbf{B})} \leq 1$, where $h(y)=|\nabla u(y)|^{p_{1}}$.

For a proof of Theorem 6.1, we need the following result, which gives an essential tool in treating monotone functions.

Lemma 6.2. (cf. $[9,10,15])$ Let $p_{1}>N-1$. If $u$ is a monotone Sobolev function on $B\left(x_{0}, 2 r\right)$, then

$$
|u(x)-u(y)|^{p_{1}} \leq M r^{p_{1}-N} \int_{B\left(x_{0}, 2 r\right)}|\nabla u(z)|^{p_{1}} d z \quad \text { whenever } x, y \in B\left(x_{0}, r\right) .
$$

Lemma 6.2 is a consequence of Sobolev's theorem, so that the restriction $p_{1}>$ $N-1$ is needed; for a proof of Lemma 6.2, see for example [9] or [15, Theorem 5.2, Chap. 8].

Now we are ready to prove Theorem 6.1 , along the same lines as in the proof of [17, Theorem 2].

Proof. Let $u$ be a monotone function on $\mathbf{B}$ such that $\|h\|_{M^{\Phi, \omega}(\mathbf{B})} \leq 1$ with $p_{1}>N-1$, where $h(y)=|\nabla u(y)|^{p_{1}}$. Let $r_{j}=2^{-j-1}$ and $t_{j}=1-r_{j-1}$ for $j=1,2, \ldots$ Using (6.1), we obtain from the proof of [17, Theorem 2] that

$$
\begin{aligned}
& \left|S_{q}\left(u, t_{j}\right)-S_{q}\left(u, t_{j+m}\right)\right| \\
& \leq C \sum_{\ell=j}^{j+m} r_{\ell}^{-\left(N-p_{1}-1\right) / p_{1}+(N-1) / q}\left(r_{\ell}^{-1} \int_{B\left(0,1-r_{\ell}\right) \backslash B\left(0,1-3 r_{\ell}\right)}|\nabla u(y)|^{p_{1}} d y\right)^{1 / p_{1}} .
\end{aligned}
$$

Hence, we have by $(\omega 6)$ and $(\Phi 3)$

$$
\begin{aligned}
& \left|S_{q}\left(u, t_{j}\right)-S_{q}\left(u, t_{j+m}\right)\right| \\
& \leq C \sum_{\ell=j}^{j+m} r_{\ell}^{-\left(N-p_{1}-1\right) / p_{1}+(N-1) / q} \Phi^{-1}\left(r_{\ell}^{-1} \int_{B\left(0,1-r_{\ell}\right) \backslash B\left(0,1-3 r_{\ell}\right)} \Phi\left(|\nabla u(y)|^{p_{1}}\right) d y\right)^{1 / p_{1}}
\end{aligned}
$$




$$
\begin{aligned}
& \leq C \sum_{\ell=j}^{j+m} r_{\ell}^{-\left(N-p_{1}-1\right) / p_{1}+(N-1) / q} \Phi^{-1}\left(r_{\ell}^{-1} \omega\left(r_{\ell}\right)^{-p}\right)^{1 / p_{1}} \\
& \leq C r_{j+m}^{-\left(N-p_{1}-1\right) / p_{1}+(N-1) / q} \Phi^{-1}\left(r_{j+m}^{-1} \omega\left(r_{j+m}\right)^{-p}\right)^{1 / p_{1}} .
\end{aligned}
$$

If $t_{j} \leq r<1$, then we take $m$ such that $t_{j+m-1} \leq r<t_{j+m}$ and establish

$$
\left|S_{q}\left(u, t_{j}\right)-S_{q}(u, r)\right| \leq C(1-r)^{-\left(N-p_{1}-1\right) / p_{1}+(N-1) / q} \Phi^{-1}\left((1-r)^{-1} \omega(1-r)^{-p}\right)^{1 / p_{1}} .
$$

Therefore it follows from $(\omega 6)$ that

$$
\limsup _{r \rightarrow 1-}(1-r)^{\left(N-p_{1}-1\right) / p_{1}-(N-1) / q} \Phi^{-1}\left((1-r)^{-1} \omega(1-r)^{-p}\right)^{-1 / p_{1}} S_{q}(u, r) \leq C,
$$

as required.

\section{References}

[1] Adams, D. R., and J. XiAo: Morrey spaces in harmonic analysis. - Ark. Mat. 50:2, 2012, 201-230.

[2] Burenkov, V. I., A. Gogatishvili, V. S. Guliyev, and R. Ch. Mustafayev: Boundedness of the fractional maximal operator in local Morrey-type spaces. - Complex Var. Elliptic Equ. 55:8-10, 2010, 739-758.

[3] Burenkov, V. I., A. Gogatishvili, V. S. Guliyev, and R. Ch. Mustafayev: Boundedness of the Riesz potential in local Morrey-type spaces. - Potential Anal. 35:1, 2011, 67-87.

[4] Gardiner, S. J.: Growth properties of $p$ th means of potentials in the unit ball. - Proc. Amer. Math. Soc. 103, 1988, 861-869.

[5] Heinonen, J., T. KilpeläInen, and O. Martio: Nonlinear potential theory of degenerate elliptic equations. - Clarendon Press, 1993.

[6] Koskela, P., J. J. Manfredi, and E. Villamor: Regularity theory and traces of $\mathcal{A}$ harmonic functions. - Trans. Amer. Math. Soc. 348, 1996, 755-766.

[7] Lebesgue, H.: Sur le probléme de Dirichlet. - Rend. Circ. Mat. Palermo 24, 1907, 371-402.

[8] Maeda, F.-Y., Y. Mizuta, and T. Shimomura: Growth properties of Musielak-Orlicz integral means for Riesz potentials. - Nonlinear Anal. 112, 2015, 69-83.

[9] Manfredi, J. J., and E. Villamor: Traces of monotone Sobolev functions. - J. Geom. Anal. $6,1996,433-444$.

[10] Manfredi, J. J., and E. Villamor: Traces of monotone Sobolev functions in weighted Sobolev spaces. - Illinois J. Math. 45, 2001, 403-422.

[11] MizutA, Y.: Spherical means of Beppo Levi functions. - Math. Nachr. 158, 1992, 241-262.

[12] Mizuta, Y.: Growth properties of $p$ th hyperplane means of Green potentials in a half space. - J. Math. Soc. Japan 44:1, 1992, 1-12.

[13] Mizuta, Y.: Continuity properties of potentials and Beppo-Levi-Deny functions. - Hiroshima Math. J. 23, 1993, 79-153.

[14] Mizuta, Y.: Tangential limits of monotone Sobolev functions. - Ann. Acad. Sci. Fenn. Math. 20, 1995, 315-326.

[15] Mizuta, Y.: Potential theory in Euclidean spaces. - Gakkōtosyo, Tokyo, 1996.

[16] Mizuta, Y.: Hyperplane means of potentials. - J. Math. Anal. Appl. 201, 1996, 226-246.

[17] Mizuta, Y., and T. Shimomura: Boundary limits of spherical means for BLD and monotone BLD functions in the unit ball. - Ann. Acad. Sci. Fenn. Math. 24, 1999, 45-60. 
[18] Morrey, C. B.: On the solutions of quasi-linear elliptic partial differential equations. - Trans. Amer. Math. Soc. 43, 1938, 126-166.

[19] Shimomura, T.: $L^{q}$-mean limits for Taylor's expansion of Riesz potentials of functions in Orlicz classes. - Hiroshima Math. J. 27:1, 1997, 159-175.

[20] Shimomura, T.: Growth properties of hyperplane integrals of Sobolev functions in a half space. - Osaka J. Math. 38, 2001, 759-773.

[21] Stoll, M.: Boundary limits of subharmonic functions in the unit disc. - Proc. Amer. Math. Soc. 93, 1985, 567-568.

[22] Stoll, M.: Rate of growth of $p$ th means of invariant potentials in the unit ball of $C^{n}$. - J. Math. Anal. Appl. 143, 1989, 480-499.

[23] Stoll, M.: Rate of growth of $p$ th means of invariant potentials in the unit ball of $C^{n}$, II. - J. Math. Anal. Appl. 165, 1992, 374-398.

[24] Villamor, E., and B. Q. Li: Analytic properties of monotone Sobolev functions. - Complex Variables Theory Appl. 46, 2001, 255-263.

[25] Vuorinen, M.: On functions with a finite or locally bounded Dirichlet integral. - Ann. Acad. Sci. Fenn. Math. 9, 1984, 177-193.

[26] Vuorinen, M.: Conformal geometry and quasiregular mappings. - Lectures Notes in Math. 1319, Springer, 1988.

Received 31 October $2016 \bullet$ Accepted 24 April 2017 